\title{
Chemical reactivity of the compressed noble gas atoms and their reactivity dynamics during collisions with protons
}

\author{
P K CHATTARAJ*, B MAITI and U SARKAR \\ Department of Chemistry, Indian Institute of Technology, \\ Kharagpur 721 302, India \\ e-mail: pkc@chem.iitkgp.ernet.in
}

MS received 18 December 2003; revised 25 April 2003

\begin{abstract}
Attempts are made to gain insights into the effect of confinement of noble gas atoms on their various reactivity indices. Systems become harder, less polarizable and difficult to excite as the compression increases. Ionization also causes similar effects. A quantum fluid density functional technique is adopted in order to study the dynamics of reactivity parameters during a collision between protons and $\mathrm{He}$ atoms in different electronic states for various projectile velocities and impact parameters. Dynamical variants of the principles of maximum hardness, minimum polarizability and maximum entropy are found to be operative.
\end{abstract}

Keywords. Confinement; reactivity dynamics; quantum fluid density functional theory.

\section{Introduction}

For some years now the influence of spatial confinement of atoms or molecules on their physical and chemical properties has been considered an important area of research. The concept of confined quantum systems ${ }^{1}$ originated from the idea of simulating the effect of pressure on an atom by confining it in an impenetrable spherical box. This is useful for understanding the behaviour of: the effect of pressure on energy levels, polarizability of atoms and molecules, ${ }^{1-19}$ semiconductor quantum dots, quantum wires, quantum wells etc. ${ }^{20}$ Physical and chemical properties of systems are highly dependent on the size and shape of the confined volume. ${ }^{20-30}$ In this article we have calculated the softness, polarizability, mean excitation energy, total energy and expectation values of $r, r^{2}$ and $1 / r$ of $\mathrm{He}$ and $\mathrm{Ne}$ at various degrees of confinement.

Density functional theory ${ }^{31}$ is successful in providing insights into the concepts of chemical reactivity parameters like hardness, polarizability, ionization energy etc. In DFT, the total energy functional for an $\mathrm{N}$-electron system can be written as

$$
E[\rho]=T[\rho]+E_{x}[\rho]+E_{c}[\rho]-\int \frac{Z}{r} \rho(\mathbf{r}) \mathrm{d} \mathbf{r}+\frac{1}{2} \iint \frac{\rho(\mathbf{r}) \rho\left(\mathbf{r}^{\prime}\right)}{\left|\mathbf{r}-\mathbf{r}^{\prime}\right|} \mathrm{d} \mathbf{r} \mathrm{d} \mathbf{r}^{\prime}
$$

We write

*For correspondence 


$$
T[\rho]=C_{k} \int \rho^{5 / 3}(\mathbf{r}) \mathrm{d} \mathbf{r}-\frac{\lambda_{1}}{8} \int \frac{\mathbf{r} \cdot \nabla \rho(\mathbf{r})}{\mathbf{r}^{2}} \mathrm{~d} \mathbf{r},
$$

is the kinetic energy functional; ${ }^{32}$

$$
\begin{aligned}
& C_{k}=(3 / 10)\left(3 \pi^{2}\right)^{2 / 3}, \\
& E_{x}=-C_{x} \int \rho^{4 / 3}(\mathbf{r}) \mathrm{d} \mathbf{r}, \\
& \lambda_{1}=1 / 5
\end{aligned}
$$

is the Dirac local exchange energy functional; ${ }^{33}$

$$
\begin{aligned}
& C_{x}=(3 / 4 \pi)\left(3 \pi^{2}\right)^{1 / 3}, \\
& E_{c}=-\int \frac{\rho(\mathbf{r})}{9 \cdot 81+21 \cdot 437 \rho^{-1 / 3}} \mathrm{~d} \mathbf{r},
\end{aligned}
$$

is a Wigner type local correlation energy functional ${ }^{34}$.

Electronegativity $(\chi)^{35}$ and hardness $(\eta)^{36,37}$ which manifest the response of the system when $N$ varies keeping while $v(\mathbf{r})$ is constant are respectively defined as

$$
\chi=-\mu=-(\partial E / \partial N)_{v(\mathbf{r})},
$$

and

$$
\eta=\frac{1}{2}\left(\partial^{2} E / \partial N^{2}\right)_{v(\mathbf{r})}=\frac{1}{2}(\partial \mu / \partial N)_{v(\mathbf{r})}
$$

In (2) and (3) $N, v(\mathbf{r})$ and $\mu$ are the total number of electrons, external potential and chemical potential respectively. Electronegativity is the power of an atom in a molecule to attract electrons to itself. ${ }^{38}$ Pearson $^{39}$ introduced the hardness concept through his hard-soft acid-base (HSAB) principle which states that 'hard likes hard and soft likes soft'. Apart from (3) hardness can also be defined as ${ }^{40}$

$$
\eta=\frac{1}{N} \iint \eta\left(\mathbf{r}, \mathbf{r}^{\prime}\right) f\left(\mathbf{r}^{\prime}\right) \rho(\mathbf{r}) \mathrm{d} \mathbf{r} \mathrm{d} \mathbf{r}^{\prime}
$$

where $f(\mathbf{r})$ and $\eta\left(\mathbf{r}, \mathbf{r}^{\prime}\right)$ are the Fukui function ${ }^{40,41}$ and hardness kernel ${ }^{42}$ respectively. The $^{2}$ Fukui function and hardness kernel are respectively defined as:

$$
f(\mathbf{r})=\left(\frac{\partial \rho(\mathbf{r})}{\partial N}\right)_{v(\mathbf{r})}=\left(\frac{\delta \mu}{\delta v(\mathbf{r})}\right)_{N},
$$


and

$$
\eta\left(\mathbf{r}, \mathbf{r}^{\prime}\right)=\frac{1}{2} \frac{\delta^{2} F[\rho]}{\delta \rho(\mathbf{r}) \delta \rho\left(\mathbf{r}^{\prime}\right)}
$$

where $F[\rho]$ is the Hohenberg-Kohn-Sham ${ }^{43,44}$ universal functional of DFT.

Collisions of noble gas atoms with protons at low, intermediate and high-projectile velocities with different impact parameters have become an important area of research for theoreticians $^{45}$ and experimentalists ${ }^{46}$ because such processes play an important role in nuclear physics and astrophysics. Theoretically, collision dynamics can be explained with the help of density functional theory $(\mathrm{DFT})^{31}$ by studying the ground- and excited-state electronic structure and properties of many-electron systems. According to DFT, the single-particle density $\rho(\mathbf{r})$ contains all the information about a system and the total energy attains a minimum value for the true $\rho(\mathbf{r})$. A time-dependent (TD) version of DFT is also available for an arbitrary TD potential whose mapping with the density has been shown to be uniquely invertible up to an additive TD function in the potential. ${ }^{47}$ This TDDFT strengthens the quantum fluid dynamics (QFD). ${ }^{48}$ For studying collision dynamics, we adopted a method, essentially an amalgamation of density functional theory (DFT) and quantum fluid dynamics (QFD), called the time-dependent quantum fluid density functional theory (TDQFDFT). Successful applications of TDQFDFT have already been made in intense laser-atom dynamics leading to photoionization ${ }^{49}$ and photoemission, $^{50}$ suppression of ionization ${ }^{51}$ and high energy $\mathrm{H}^{+}-\mathrm{Ne}$ and $\mathrm{H}^{+}-\mathrm{He}$ collisions. ${ }^{52}$ We hope that these will lead to extensive applications of TDQFDFT to molecular dynamics, e.g. dissociation of molecules by an external field where the calculation of both nuclear and electronic motions ought to be considered. This adopts an impulse approximation, i.e., a straight-line trajectory for the projectile. In this article, we study the reactivity dynamics at different velocities and impact parameters. It is also important to know how the atom would response to the collision with a proton so far as its reactivity is concerned. The behaviour of the helium atom on collision with the proton can be explained with different reactivity parameters like electronegativity, hardness, polarizability, entropy, electrophilicity and nucleophilicity indices and uncertainty product. During the collision process, polarizability $(\alpha)$ is the corresponding response due to a change in $v(\mathbf{r})$ for constant $N$. A Shannon-type entropy $(S)$ was introduced by Deb and Chattaraj ${ }^{53}$ within a quantum fluid density functional framework. During molecule formation the electronegativity of the pertinent atoms get equalized. ${ }^{54} \mathrm{~A}$ stable configuration or a favourable process is generally associated with maximum hardness $(\mathrm{MH}){ }^{37}$ minimum polarizability $(\mathrm{MP})^{55}$ and maximum entropy $(\mathrm{ME})^{56}$ values. The conditions for maximum hardness and entropy as well as minimum polarizability complement the usual minimum energy criterion for stability. Recently Parr et $a l^{57}$ have defined the electrophilicity index $(W)$. We also study here the behaviour of $(1 / W)$, a valid candidate for the nucleophilicity index. It has also been shown recently ${ }^{58}$ that the uncertainty product or the phase space volume $\left(V_{p s}\right)$ is a measure of quantum fluctuations and hence has a bearing on the study of quantum domain behaviour of classically chaotic systems.

The theoretical background of the present work is provided in $\S 2$. Section 3 contains the numerical details, while results and discussions are given in $\S 4$. Finally, $\S 5$ presents some concluding remarks. 


\section{Theoretical background}

The polarizability $(\alpha),{ }^{59,60}$ which is a measure of the response of the system by varying the external potential $(v(\mathbf{r}))$ keeping the total number of electrons $(N)$ constant, is calculated form the relation

$$
\alpha=\left(\frac{4 \pi}{3}\right) \int_{0}^{\infty} s(r) r^{4} \mathrm{~d} r
$$

The mean excitation energy ${ }^{61}(I)$ in the local plasma approximation of charge distribution $^{62}$ is defined as follows:

$$
I=2 \sqrt{\pi} \gamma \exp \left[-S_{\rho} / 2 Z\right]
$$

where $S_{\rho}$ is the Shannon entropy and $Z$ is the number of electrons of the atom. The chemical shift factor $\gamma$ varies between 1 and $\sqrt{2}$.

The hardness kernel $\eta\left(\mathbf{r}, \mathbf{r}^{\prime}\right),(6)$, is calculated using the following local form for $F[\rho]$,

$$
F[\rho]=T^{\text {local }}[\rho]+V_{e e}^{\text {local }}[\rho]
$$

where the local kinetic energy ${ }^{63}$ and electron-electron repulsion energy ${ }^{64}$ are taken as

$$
T^{\text {local }}[\rho]=c_{k} \int \rho^{5 / 3} \mathrm{~d} \mathbf{r}+c_{x} \int \frac{\rho^{4 / 3} / \mathbf{r}}{1+\left(r \rho^{1 / 3} / 0 \cdot 043\right)} \mathrm{d} \mathbf{r},
$$

and

$$
V_{e e}^{\text {local }}[\rho]=0 \cdot 7937(N-1)^{2 / 3} \int \rho^{4 / 3} \mathrm{~d} \mathbf{r} .
$$

These local functionals are used because of the simplicity in the calculation of the second-order functional derivative and the associated Fukui function within this local model.

The dynamical polarizability is written as

$$
\alpha(t)=\left|D_{\text {ind }}^{z}(t)\right| /\left|F_{z}(t)\right|
$$

where $D_{\text {ind }}^{z}(t)$ is the electronic part of the induced dipole moment given as,

$$
D_{i n d}^{z}(t)=\int z \rho(\mathbf{r}, t) \mathrm{d} \mathbf{r},
$$

and $F_{z}(t)$ is the $z$-component of the external Coulomb field due to the incoming proton.

The TD entropy is defined as, 


$$
S(t)=\int\left\{\frac{5}{2}-\ln \rho(\mathbf{r}, t)+\frac{3}{2} \ln (k \theta(\mathbf{r}, t) / 2 \pi)\right\} k \rho(\mathbf{r}, t) \mathrm{d} \mathbf{r},
$$

where $k$ is the Boltzmann constant and $\theta(\mathbf{r}, t)$ is a space-time dependent temperature given in terms of the kinetic energy density $t_{s}(\mathbf{r}, \rho(\mathbf{r}, t))$ as

$$
t_{s}(\mathbf{r} ; \rho(\mathbf{r}, t))=\frac{3}{2} k \theta(\mathbf{r}, t) \rho(\mathbf{r}, t)+\left[|\mathbf{j}(\mathbf{r}, t)|^{2} / 2 \rho(\mathbf{r}, t)\right]
$$

The electrophilicity index $(W)$ is defined as

$$
W=\mu^{2} / 2 \eta
$$

The term $(1 / W)$ is called the nucleophilicity index.

The phase space volume or the uncertainty product, $V_{p s}{ }^{58,59}$ has been shown to be an important diagnostic of the quantum signature of classical chaos as related to the compactness of the electron cloud. For the present problem it may be defined as

$$
\begin{array}{r}
V_{p s}=\left\{\left\langle\left(p_{\tilde{\rho}}-\left\langle p_{\tilde{\rho}}\right\rangle\right)^{2}\right\rangle\left\langle\left(p_{z}-\left\langle p_{z}\right\rangle\right)^{2}\right\rangle\right. \\
\left.\left\langle(\tilde{\rho}-\langle\tilde{\rho}\rangle)^{2}\right\rangle\left\langle(z-\langle z\rangle)^{2}\right\rangle\right\}^{1 / 2} .
\end{array}
$$

A sharp increase in $V_{p s}(t)$ signals chaotic motion since it is a measure of the associated quantum fluctuations.

The dynamics of a quantum system are described in terms of the flow of a probability fluid associated with the probability density $\rho(\mathbf{r})$ and the current density $\mathbf{j}(\mathbf{r})$. The time evolution of these two quantities are governed by two basic QFD equations ${ }^{53}$, viz. the equation of continuity,

$$
(\partial \rho / \partial t)+\nabla \cdot(\rho \nabla \xi)=0
$$

and the equation of motion,

$$
\frac{\partial \xi}{\partial t}+\frac{1}{2}(\nabla \xi)^{2}+\frac{\delta G[\rho]}{\delta t}+\int \frac{\rho(\mathbf{r}, t)}{\left|\mathbf{r}-\mathbf{r}^{\prime}\right|} \mathrm{d} \mathbf{r}^{\prime}+v_{\mathrm{ext}}(\mathbf{r}, t)=0,
$$

where $\xi$ is the velocity potential. The universal functional $G[\rho]$ comprises kinetic and exchange correlation energy functionals and $v_{\text {ext }}(\mathbf{r}, t)$ is the external potential. Atomic units are used throughout this article unless otherwise specified.

A three-dimensional complex-valued hydrodynamical function $\phi(\mathbf{r}, t)$ can be defined in the following polar form:

$$
\begin{aligned}
& \phi(\mathbf{r}, t)=\rho(\mathbf{r}, t)^{1 / 2} \exp [i \xi(\mathbf{r}, t)], \\
& \rho(\mathbf{r}, t)=|\phi(\mathbf{r}, t)|^{2},
\end{aligned}
$$




$$
\mathbf{j}(\mathbf{r}, t)=\left[\phi_{r e} \nabla \phi_{i m}-\phi_{i m} \nabla \phi_{r e}\right]=\rho \nabla \xi .
$$

A quantum fluid density functional theory (QFDFT) $)^{53}$ was developed to study the time evolution of $\phi(\mathbf{r}, t)$ by combining (14) to generate the following generalized nonlinear Schrödinger equation (GNLSE) in cylindrical polar coordinates $(0<\tilde{\rho} \leq \infty$; $-\infty \leq \tilde{z} \leq \infty ; 0 \leq \tilde{\phi} \leq 2 \pi)$,

$$
\begin{aligned}
& {\left[\frac{1}{2} \nabla_{\tilde{\rho}, \tilde{z}}^{2}+V_{\mathrm{eff}}\left(\rho ; \tilde{\rho}, \tilde{z}, \tilde{\rho}_{p}, \tilde{z}_{p}, t\right)\right] \phi\left(\tilde{\rho}, \tilde{z}, \tilde{\rho}_{p}, \tilde{z}_{p}, t\right)} \\
& \quad=i \frac{\partial}{\partial t} \phi\left(\tilde{\rho}, \tilde{z}, \tilde{\rho}_{p}, \tilde{z}_{p}, t\right), \quad i=\sqrt{-1}, \\
& V_{\mathrm{eff}}\left(\rho ; \tilde{\rho}, \tilde{z}, \tilde{\rho}_{p}, \tilde{z}_{p}, t\right)=\frac{\delta T_{N W}}{\delta \rho}+\frac{\delta E_{x c}}{\delta \rho}+\int \frac{\rho\left(\mathbf{r}^{\prime}, t\right)}{\left|\mathbf{r}-\mathbf{r}^{\prime}\right|}+v_{\mathrm{ext}}(\mathbf{r}, t),
\end{aligned}
$$

where $T_{N W}$ and $E_{x c}$ denote the non-Weizsäcker part of the kinetic energy and exchange correlation energy functionals respectively. To construct the effective potential (17), of (16), we need $T_{N W}, E_{x c}$ and $v_{\text {ext }}(\mathbf{r}, t)$. The explicit form for $E_{x c}$ written as

$$
E_{x c}[\rho]=E_{x}[\rho]+E_{c}[\rho],
$$

where $E_{x}$ is a modified Dirac exchange functional: $:^{63}$

$$
E_{x}[\rho]=-c_{x}\left[\int \rho^{4 / 3}+\int \frac{\rho^{4 / 3} \mathrm{~d} \mathbf{r}}{1+\left(\mathbf{r}^{2} \rho^{2 / 3} / 0 \cdot 0244\right)} \mathrm{d} \mathbf{r}\right] ;
$$

and $E_{c}[\rho]$ is a Wigner-type parametrized correlation energy functional given by

$$
E_{c}[\rho]=-\int \frac{\rho}{9.81+21.437 \rho^{-1 / 3}} \mathrm{dr} .
$$

The kinetic energy functional for this problem is taken as

$$
T[\rho]=c_{k} \int \rho^{5 / 3} \mathrm{~d} \mathbf{r}+\frac{1}{8} \int \frac{\nabla \rho . \nabla \rho}{\rho} \mathrm{d} \mathbf{r}-a(N) \lambda \int \frac{\rho^{4 / 3} / \mathbf{r}}{1+\left(\mathbf{r} \rho^{1 / 3} / 0 \cdot 043\right)} \mathrm{d} \mathbf{r},
$$

where

$$
\begin{aligned}
& \lambda=30(3 / \pi)^{1 / 3}, \\
& a(N)=a_{0}+a_{1} N^{-1 / 3}+a_{2} N^{-2 / 3}, \\
& a_{0}=0 \cdot 1279, \quad a_{1}=0 \cdot 1811, \quad a_{2}=-0 \cdot 0819 .
\end{aligned}
$$


For studying the collision process between He atoms and protons the whole scattering system is considered a supermolecule and thus the corresponding kinetic energy functional comprises two parts: ${ }^{.8}$

$$
T[\rho]=T_{a t}[\rho]+T_{m o l}[\rho]
$$

where the atomic part $T_{a t}[\rho]$ is taken as given in (18) and $T_{m o l}[\rho]$ is given by

$$
T_{m o l}[\rho]=\iint \frac{1}{N^{2}}\left[\frac{1}{R^{12}}-\left(\frac{N}{10}\right)^{14} R^{2} \exp (-0 \cdot 8 R)\right] \rho(\mathbf{r}) \rho\left(\mathbf{r}^{\prime}\right) \mathrm{d} \mathbf{r} \mathrm{d} \mathbf{r}^{\prime},
$$

where $R$ is the internuclear distance.

The form of $v_{e x t}(\mathbf{r}, t)$ for the problem is taken as

$$
v_{e x t}(\mathbf{r}, t)=-\frac{2}{\left|R_{1}(t)-\mathbf{r}\right|}-\frac{1}{\left|R_{2}(t)-\mathbf{r}\right|^{\prime}},
$$

where $R_{1}$ and $R_{2}$ are radius vectors of the target (He nucleus) and the projectile $\left(\mathrm{H}^{+}\right)$ respectively. The position of the target nucleus is chosen as the origin of the coordinate system and that of the projectile is determined by a classical trajectory. ${ }^{65}$ The trajectory of the projectile is determined with the help of a classical equation of motion which is given by

$$
M_{P} \frac{\mathrm{d}^{2} R_{2}}{\mathrm{~d} t^{2}}=-\nabla_{\mathbf{R}_{2}} \cup\left[E_{P}, \rho\left(\mathbf{R}_{1}, \mathbf{R}_{2}, t\right) ; Z_{T} Z_{P}, t\right]
$$

where

$$
\mathbf{R}_{1}=\left(\tilde{\rho}^{2}+\tilde{z}^{2}\right)^{1 / 2},
$$

and

$$
\mathbf{R}_{2}=\left(\tilde{\rho}_{P}^{2}(t)+\tilde{z}_{P}^{2}(t)\right)^{1 / 2}
$$

Here $M_{P}$ is the mass of the projectile, $E_{P}$, its kinetic energy, $Z_{P}$ and $Z_{T}$ are nuclear charges of the projectile and target respectively. Relativistic contributions are not taken into account in this work. In the cylindrical coordinate system, (22a) can be written in compact matrix form as,

$$
M_{P} \frac{\mathrm{d}^{2}}{\mathrm{~d} t^{2}}\left(\begin{array}{c}
\tilde{\rho}_{P} \\
\tilde{z}_{P}
\end{array}\right)=-\left(\begin{array}{cc}
\frac{\partial}{\partial \tilde{\rho}_{P}} & \frac{\partial}{\partial \tilde{\rho}_{P}} \\
\frac{\partial}{\partial \tilde{z}_{P}} & \frac{\partial}{\partial \tilde{z}_{P}}
\end{array}\right)\left(\begin{array}{c}
\mathrm{U}_{1} \\
\mathrm{U}_{2}
\end{array}\right),
$$

where

$$
\mathrm{U}_{1}=Z_{P} Z_{T} /\left(\tilde{\rho}_{P}+\tilde{z}_{P}\right)^{1 / 2}
$$

and

$$
\bigcup_{2}=\int V_{\mathrm{eff}}\left(\mathbf{r}_{P}, \mathbf{r}, t\right) \mathrm{d} \mathbf{r}
$$


For the evolution of the first derivative with respect to $\tilde{z}_{P}$ and $\tilde{\rho}_{P}$ as well as the form of $V_{\text {eff }}$ given by (17), (23a) can be simplified into two equations, viz.

$$
\begin{aligned}
M_{P} \frac{\mathrm{d}^{2} \tilde{\rho}_{P}}{\mathrm{~d} t^{2}} & =-\frac{Z_{P} Z_{T} \tilde{\rho}_{P}}{\left(\tilde{\rho}_{P}+\tilde{z}_{P}\right)^{3 / 2}} \\
& -Z_{P} \int\left[\int \rho(\tilde{\rho}, \tilde{z}, t) J_{0}(k \tilde{\rho}) J_{1}\left(k \tilde{\rho}_{P}\right) \exp \left(-k\left|\tilde{z}-\tilde{z}_{P}\right|\right) \mathrm{d} k\right] \mathrm{d} \mathbf{r}
\end{aligned}
$$

and

$$
\begin{aligned}
M_{P} \frac{\mathrm{d}^{2} \tilde{z}_{P}}{\mathrm{~d} t^{2}} & =-\frac{Z_{P} Z_{T} \tilde{z}_{P}}{\left(\tilde{\rho}_{P}+\tilde{z}_{P}\right)^{3 / 2}} \\
& +Z_{P} \int\left[\int \rho(\tilde{\rho}, \tilde{z}, t) J_{0}(k \tilde{\rho}) J_{0}\left(k \tilde{\rho}_{P}\right) \exp \left(-k\left|\tilde{z}-\tilde{z}_{P}\right|\right)\{ \pm k \mathrm{~d} k\}\right] \mathrm{d} \mathbf{r} .
\end{aligned}
$$

In (25), the '-' sign is taken if $\tilde{z}-\tilde{z}_{P}>0$ and the ' + ' sign is taken if $\tilde{z}-\tilde{z}_{P}<0$. In (24) and (25), $J_{0}$ and $J_{1}$ are the zeroth-order and first-order Bessel functions respectively. The finite-difference (central) forms of (24) and (25) can be written as

$$
\tilde{\rho}_{P}^{n+1}=2 \tilde{\rho}_{P}^{n}-\tilde{\rho}_{P}^{n-1}+F_{\tilde{\rho}_{P}}^{n}(\mathrm{~d} t)^{2} / M_{P},
$$

and

$$
\tilde{z}_{P}^{n+1}=2 \tilde{z}_{P}^{n}-\tilde{z}_{P}^{n-1}+F_{\tilde{z}_{P}}^{n}(\mathrm{~d} t)^{2} / M_{P}
$$

where the index $n$ defines the discretized time domain. $F_{\tilde{\rho}_{P}}^{n}$ on the right-hand side of (25) is given by

$$
\begin{aligned}
F_{\tilde{\rho}_{P}}^{n} & =-\frac{Z_{P} Z_{T} \tilde{\rho}_{P}^{n}}{\left\{\left(\tilde{\rho}_{P}^{n}\right)^{2}+\left(\tilde{z}_{P}^{n}\right)^{2}\right\}^{3 / 2}} \\
& -Z_{P} \int\left[\int \rho(\tilde{\rho}, \tilde{z}, t) J_{0}(k \tilde{\rho}) k J_{1}\left(k \tilde{\rho}_{P}^{n}\right) \exp \left(-k\left|\tilde{z}-\tilde{z}_{P}^{n}\right|\right) \mathrm{d} k\right] 2 \pi \tilde{\rho} \mathrm{d} \tilde{\rho} \mathrm{d} \tilde{z},
\end{aligned}
$$

while $F_{\tilde{z}_{P}}^{n}$ on the right-hand side of (26) is given by

$$
\begin{aligned}
F_{\tilde{\rho}_{P}}^{n} & =-\frac{Z_{P} Z_{T} \tilde{z}_{P}^{n}}{\left\{\left(\tilde{\rho}_{P}^{n}\right)^{2}+\left(\tilde{z}_{P}^{n}\right)^{2}\right\}^{3 / 2}} \\
& -Z_{P} \int\left[\int \rho(\tilde{\rho}, \tilde{z}, t) J_{0}(k \tilde{\rho}) k J_{1}\left(k \tilde{\rho}_{P}^{n}\right) \exp \left(-k\left|\tilde{z}-\tilde{z}_{P}^{n}\right|\right)\{ \pm k \mathrm{~d} k\}\right] 2 \pi \tilde{\rho} \mathrm{d} \tilde{\rho} \mathrm{d} \tilde{z}
\end{aligned}
$$

Equations (26) and (27) indicate that in order to obtain the projectile's position $\left(\tilde{\rho}_{P}^{n+1}, \tilde{z}_{P}^{n+1}\right)$ at any advanced $(n+1)$ th time step from the nth time step, one needs the 
following quantities: (i) the electron density $\rho\left(\tilde{\rho}, \tilde{z}, \tilde{\rho}_{P}^{n}, \tilde{z}_{P}^{n}, t\right)$, (ii) the projectile's position at the nth time step $\left(\tilde{\rho}_{P}^{n}, \tilde{z}_{P}^{n}\right)$, and (iii) the projectile position at the $(n-1)$ th time step $\left(\tilde{\rho}_{P}^{n-1}, \tilde{z}_{P}^{n-1}\right)$.

The electron density of the colliding system for any position $\left(\tilde{\rho}_{P}^{n}, \tilde{z}_{P}^{n}\right)$ of the projectile can be calculated by solving (16). At any time, the projectile positions at $n$th and $(n-1)$ th time steps can be obtained from the knowledge of the same at the first and second time steps. The projectile's position at the first time step $(t=0)$ is taken as

$$
\tilde{z}_{P}^{1}=-\tilde{z}_{P}^{0} ; \quad \tilde{\rho}_{P}^{1}=b,
$$

where $b$ is the impact parameter. While that at the second time step $(t=\Delta t)$ is taken as

$$
\begin{aligned}
& \tilde{z}_{P}^{2}=-\tilde{z}_{P}^{1}+v_{P} \cos v_{0} \Delta t, \\
& \tilde{\rho}_{P}^{2}=-\tilde{\rho}_{P}^{1}+v_{P} \sin v_{0} \Delta t .
\end{aligned}
$$

Here $v_{P}$ is the projectile's incident velocity and $v_{0}$ is the scattering angle.

$$
v_{P}=\left(2 E_{P} / M_{P}\right)^{1 / 2} .
$$

Thus over a range of time the realistic path of the projectile at any incident energy can be calculated. The integrals appearing in (28) and (29) have been calculated by the trapezoidal method for the $\widetilde{\rho}^{\prime}$ and $\widetilde{z}^{\prime}$ variables, while a three-point Gauss quadrature has been employed for the variables $k$.

The validity of TDDFT allows us to legitimately assume that the mapping $v_{\mathrm{ext}}(\mathbf{r}, t) \rightarrow$ $\rho(\mathbf{r}, t)$ and $v_{\text {ext }}(\mathbf{r}, t) \rightarrow \mathbf{j}(\mathbf{r}, t)$ are uniquely invertible of $\rho(\mathbf{r}, t)$ and $\mathbf{j}(\mathbf{r}, t)$. Therefore in this context we can define a time-dependent energy quantity, $E(t)$ as a density functional as follows:

$$
\begin{aligned}
E(t) & =\frac{1}{2} \int \rho(\mathbf{r}, t)|\nabla \xi|^{2} \mathrm{~d} \mathbf{r}+T[\rho]+\frac{1}{2} \iint \frac{\rho(\mathbf{r}, t) \rho\left(\mathbf{r}^{\prime}, t\right)}{\left|\mathbf{r}-\mathbf{r}^{\prime}\right|} \mathrm{d} \mathbf{r} \mathrm{d} \mathbf{r}^{\prime} \\
& +E_{x c}[\rho]+\int v_{\mathrm{ext}}(\mathbf{r}, t) \rho(\mathbf{r}, t) \mathrm{d} \mathbf{r} .
\end{aligned}
$$

A similar time-dependent energy functional was defined within a harmonic timedependent perturbation by Deb and Ghosh ${ }^{66}$ as well as by Bartolotti. ${ }^{67}$ Runge and Gross ${ }^{47}$ defined the same for a general time-dependent problem.

Now if there exists a point $r_{c}$ where the sum of functional derivatives of total kinetic and exchange-correlation energies is equal to zero, the time-dependent chemical potential becomes equal to the total electrostatic potential at that point, that is:

$$
-\chi(t)=\mu(t)=\int \frac{\rho(\mathbf{r}, t)}{\left|\mathbf{r}_{c}-\mathbf{r}\right|} \mathrm{d} \mathbf{r}+v_{\mathrm{ext}}\left(r_{c}, t\right)
$$

where $r_{c}$ is the point at which the following condition is satisfied at that time step: 


$$
\frac{1}{2}(\nabla \xi)^{2}+\frac{\delta T}{\delta \rho}+\frac{\delta E_{x c}}{\delta \rho}=0
$$

Since $j(\mathbf{r}, 0)=0$, the above condition at $t=0$ becomes identical to that of the ground state DFT given by Politzer et $a l^{68}$ who showed through application of the electronegativity equalization principle that $r_{c}$ values provide very good estimates of the covalent radii of the atom.

\section{Computational details}

Total energy is calculated from (1). The first term of (1) represents kinetic energy and the remaining terms represent potential energy. Numerical calculation of the self-consistentfield (SCF) electronic wavefunction has been done by using the standard Herman and Skillman ${ }^{69}$ program. Since the wavefunction must vanish at the confining boundary, we have introduced this confinement condition by multiplying the un-normalized SCF wavefunction by a step function in each iteration. The step function taken here is given by $^{70}$

$$
\Theta=\exp \left[-(r / R)^{20}\right]
$$

The GNLSE was solved numerically using a leap-frog type of finite difference scheme. Azimuthal symmetry of the physical system allows us to analytically integrate over $0 \leq$ $\tilde{\phi} \leq 2 \pi$ in a cylindrical polar coordinate $(\tilde{\rho}, \tilde{\phi}, z)$ system. Equation (16) was transformed as

$$
\left\{\left(\frac{3}{4 x^{3}}\right) \frac{\partial y}{\partial x}-\left(\frac{1}{4 x^{2}}\right) \frac{\partial^{2} y}{\partial x^{2}}-\frac{\partial^{2} y}{\partial z^{2}}\right\}-\left(\frac{1}{x^{4}}-2 v_{\text {eff }}\right) y=2 i \frac{\partial y}{\partial t}
$$

where

$$
y=\tilde{\rho} \phi
$$

and

$$
\tilde{\rho}=x^{2}
$$

A detailed discussion on the numerical solution can be found elsewhere. ${ }^{55}$ The numerical solution is launched with near-Hartree-Fock density of helium in the ${ }^{1} S$ ground state ${ }^{71}$ and a ${ }^{1} P$ excited state ${ }^{72}$ of the $1 s 2 p$ electronic configuration. The temporal mesh size is taken as $\Delta t=0.025$ a.u. Different spatial grid sizes are chosen for the ground and excited states; we take $\Delta x=\Delta z=0.5$ a.u. and $\Delta x=\Delta z=0.036$ a.u. respectively. Large domains for $x$ and $z$ are taken when the excited-state density is used. Three different initial velocities of the projectile are $v_{P}=0 \cdot 1,1 \cdot 0$ and 10.0. Two different values of $b$ are also taken as $0 \cdot 6$ and $1 \cdot 2$. The values of scattering angle $\left(v_{0}\right)=0 \cdot 0$. The value of $M_{P}$ is 1836 a.u.

\section{Results and discussions}

Variation of softness with respect to cut-off radius $R$ is shown in figure 1. A decrease of cut-off radius decreases the value of softness implies the fact that the system becomes 
harder. The relative order of softness for $\mathrm{He}, \mathrm{He}^{+}, \mathrm{Ne}$ and $\mathrm{Ne}^{+}$is $S_{\mathrm{He}^{+}}<S_{\mathrm{He}}<S_{\mathrm{Ne}^{+}}<S_{\mathrm{Ne}}$, as expected. Atoms are softer than the corresponding ions.

Figure 2 presents the variation of polarizability $(\alpha)$ as a function of $R$. Polarizability is high for large values of $R$ and decreases gradually as we decrease $R$. Polarizability or softness attains a saturation value for large values of $R$. The relative order of polarizability is same as softness. Polarizability decreases on ionization.

In figure 3 we present softness vs $\alpha^{1 / 3}$. It is clear from the figure that all curves are near-straight lines (regression coefficients are given in appropriate positions). For a confined system, the linear relation between $S$ and $\alpha^{1 / 3}$ for $\mathrm{He}, \mathrm{He}^{+}, \mathrm{Ne}$ and $\mathrm{Ne}^{+}$, as would have been expected ${ }^{73-77}$ for atoms, molecules and clusters, is shown here for the first time.

Figure 4 depicts the plot of ionization energy $(I)$ versus cut-off radius for $\mathrm{He}, \mathrm{He}^{+}, \mathrm{Ne}$ and $\mathrm{Ne}^{+}$. From this figure it is clear that the increase of cut-off radius corresponds to a decrease in their mean excitation energy which is consistent with the expectation. ${ }^{78}$ The $I$ values for $\mathrm{He}$ and $\mathrm{Ne}$ are 35.390095 and 114.790594 respectively (for $R=10 \cdot 0$ ) which are very close to the values obtained by Hồ et al. ${ }^{62}$ Ions have higher $I$ values than the corresponding atoms for all $R$ values.

Figure 5 manifests how kinetic energy, potential energy and total energy change with radius. Kinetic energy increases more rapidly than potential energy with the decrease of cut-off radius. These plots are similar to those given by $\operatorname{Gimarc}^{79}$ for He atom. The kinetic energy, potential energy and total energy values, given in table 1 which are very close to those obtained by Fischer ${ }^{80}$ for free atoms $(R=10 \cdot 0)$. For large $I$, virial theorem is satisfied in both the cases.

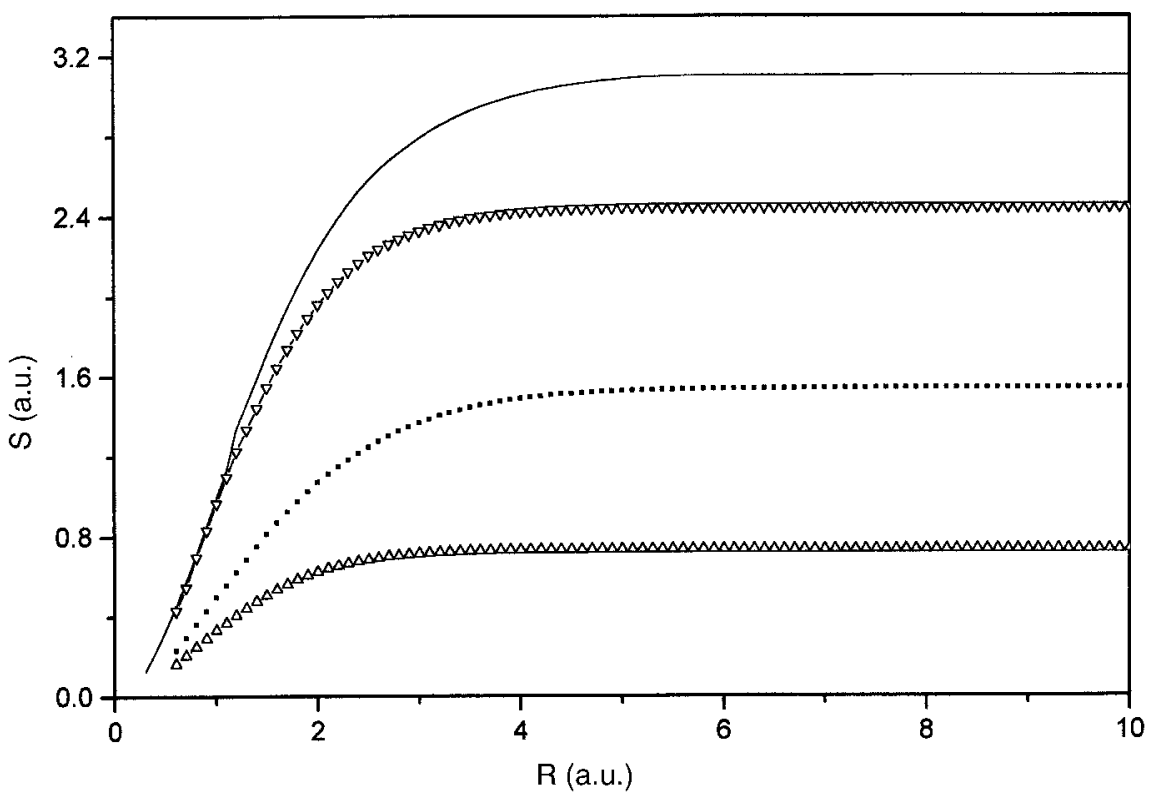

Figure 1. Plot of softness $(S$, a.u.) versus cut-off radius $(R$, a.u.) for $\mathrm{He}$ and $\mathrm{Ne}$ atoms and their ions confined in a spherical box. (…) He atom, $(-\triangle-) \mathrm{He}^{+}$ion, $(-) \mathrm{Ne}$ atom, $(-\nabla-) \mathrm{Ne}^{+}$ion. 


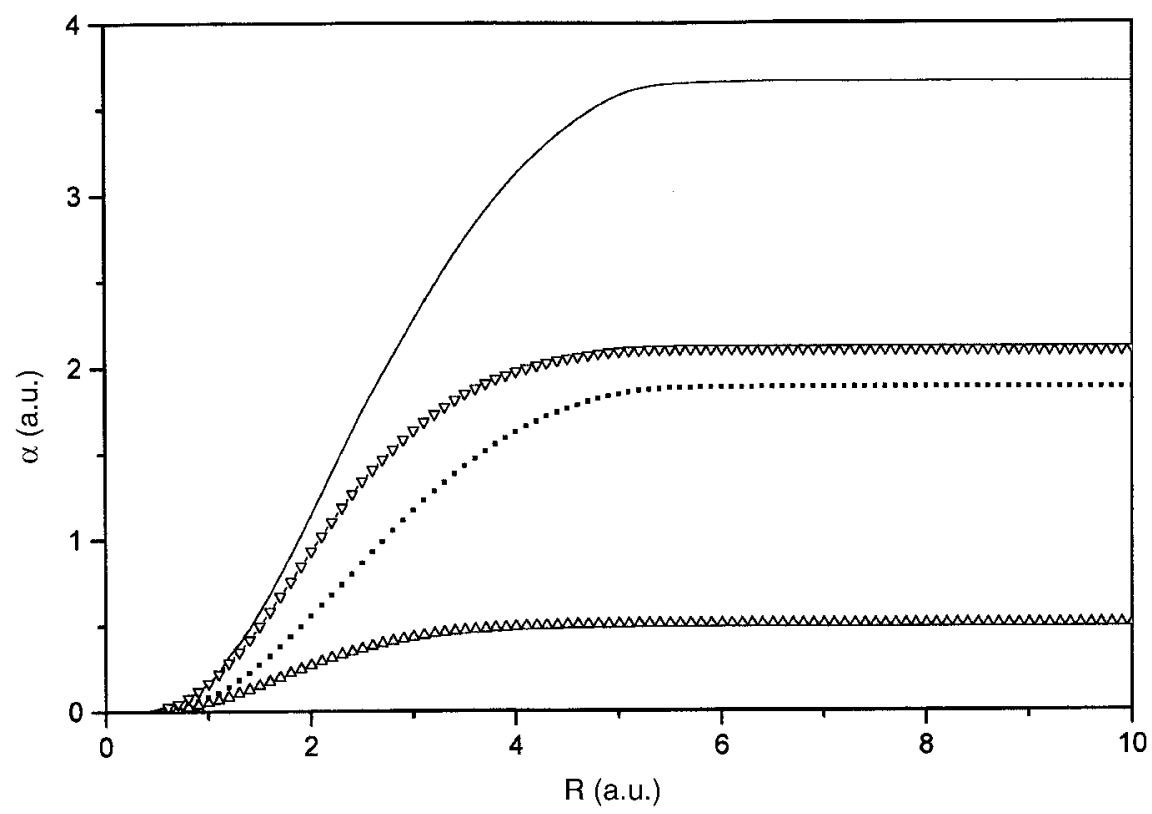

Figure 2. Plot of polarizability ( $\alpha$, a.u.) versus cut-off radius ( $R$, a.u.) for $\mathrm{He}$ and $\mathrm{Ne}$ atoms and their ions confined in a spherical box. $(\cdots \cdots) \mathrm{He}$ atom, $(-\triangle-) \mathrm{He}^{+}$ ion, $(-) \mathrm{Ne}$ atom, $(-\nabla-) \mathrm{Ne}^{+}$ion.

Expectation values of $r, r^{2}$ and $1 / r$ are listed in table 2 for total electronic charge density. Expectation values of these quantities for free $(R=10$ a.u.) He atoms are in good agreement with those of Fischer. ${ }^{81}$ Expectation values of $r$ and $r^{2}$ decrease with decrease of $R$ whereas expectation value of $1 / r$ increases with decrease of $R$ for both atoms and ions.

Evolution with time of different reactivity parameters is depicted in figures 6-12. All quantities are in atomic units. Unless otherwise specified, in all the figures, GS and ES refer to the ground and excited states of the helium atom respectively. Two different impact parameters and three different projectile velocities corresponding to $b=0 \cdot 6,1 \cdot 2$ and $v_{P}=0 \cdot 1,1 \cdot 0,10 \cdot 0$ are shown separately.

Figure 6 depicts the time-dependence of the chemical potential with different impact parameters $(b)$ and different projectile velocities $\left(\nu_{P}\right)$ in the collision process. Three distinct zones are discernible for the whole collision process: approach, encounter and departure. The concept of three collisional regimes was originally reported in terms of the time-dependent difference density and induced dipole moment profiles. ${ }^{52}$ Since nowhere in space is condition (33b) satisfied in the encounter regime, neither $r_{c}$ nor $\mu$ is calculable. After the initial transients leave, $\mu$ becomes more or less stable at the approach regime. Towards the end of the approach regime and the beginning of the departure regime $\mu$ changes drastically due to the rapid charge oscillations. These time steps bracket the encounter regime where the electron density is shared by both nuclei. While $\mu$ in these time steps is mainly negative for the ground state, it becomes both positive and negative 


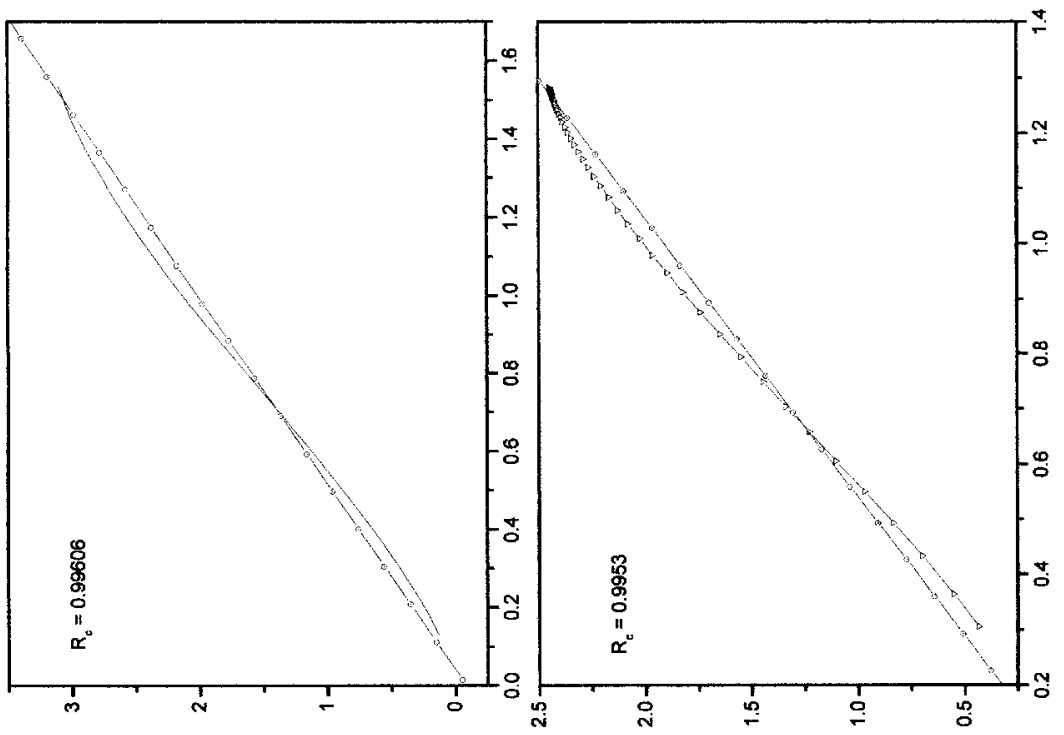

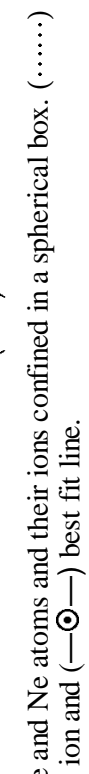

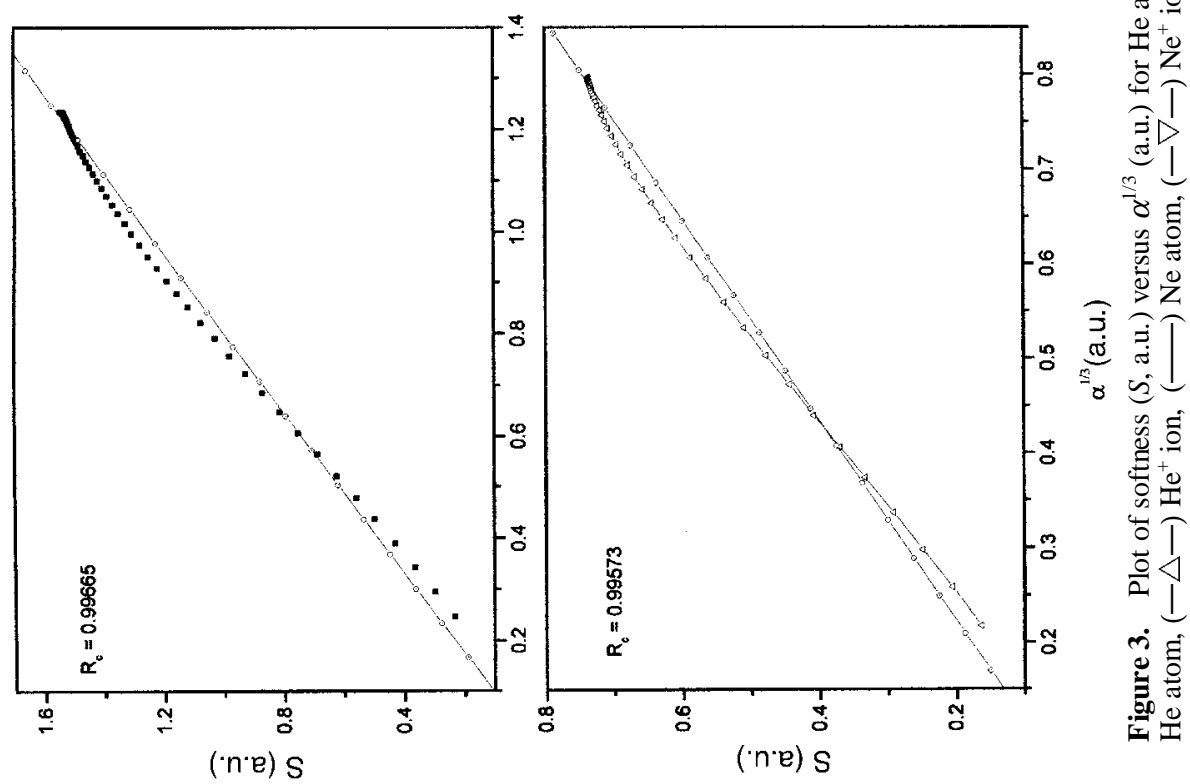


Table 1. Variation of potential, kinetic and total energies (in a.u.) versus the cutoff radius $(R$, a.u. $)$ for $\mathrm{He}$ and $\mathrm{Ne}$ atoms and theirs ions.

\begin{tabular}{|c|c|c|c|c|}
\hline Atom/ion & $R$ & Potential energy & Kinetic energy & Total energy \\
\hline \multirow[t]{12}{*}{$\mathrm{He}$} & $10 \cdot 0$ & $-5 \cdot 5854$ & 2.6794 & $-2 \cdot 9060$ \\
\hline & $8 \cdot 0$ & $-5 \cdot 5854$ & $2 \cdot 6794$ & $-2 \cdot 9060$ \\
\hline & $6 \cdot 0$ & $-5 \cdot 5854$ & $2 \cdot 6794$ & $-2 \cdot 9060$ \\
\hline & $4 \cdot 0$ & $-5 \cdot 5880$ & 2.6827 & $-2 \cdot 9052$ \\
\hline & $3 \cdot 0$ & $-5 \cdot 6067$ & 2.7077 & $-2 \cdot 8990$ \\
\hline & $2 \cdot 0$ & $-5 \cdot 7531$ & $2 \cdot 9062$ & $-2 \cdot 8470$ \\
\hline & 1.5 & $-6 \cdot 0843$ & $3 \cdot 4048$ & -2.6795 \\
\hline & 1.0 & $-7 \cdot 1464$ & $5 \cdot 1804$ & $-1 \cdot 9660$ \\
\hline & 0.8 & $-8 \cdot 1288$ & $7 \cdot 2270$ & $-0 \cdot 9018$ \\
\hline & 0.6 & -9.9263 & 11.8669 & 1.9407 \\
\hline & $0 \cdot 4$ & $-14 \cdot 4348$ & $25 \cdot 7071$ & $11 \cdot 2723$ \\
\hline & $0 \cdot 3$ & $-18 \cdot 3581$ & $44 \cdot 5086$ & $26 \cdot 1504$ \\
\hline \multirow[t]{12}{*}{$\mathrm{He}^{+}$} & $10 \cdot 0$ & $-3 \cdot 8010$ & $1 \cdot 2274$ & $-2 \cdot 5736$ \\
\hline & $8 \cdot 0$ & $-3 \cdot 8010$ & 1.2274 & $-2 \cdot 5736$ \\
\hline & $6 \cdot 0$ & $-3 \cdot 8010$ & 1.2274 & $-2 \cdot 5736$ \\
\hline & $4 \cdot 0$ & $-3 \cdot 8011$ & $1 \cdot 2275$ & $-2 \cdot 5736$ \\
\hline & $3 \cdot 0$ & $-3 \cdot 8045$ & 1.2298 & $-2 \cdot 5747$ \\
\hline & $2 \cdot 0$ & $-3 \cdot 8603$ & 1.2701 & $-2 \cdot 5902$ \\
\hline & 1.5 & $-4 \cdot 0332$ & 1.4062 & $-2 \cdot 6270$ \\
\hline & 1.0 & $-4 \cdot 6412$ & 1.9574 & $-2 \cdot 6838$ \\
\hline & $0 \cdot 8$ & $-5 \cdot 2376$ & $2 \cdot 6118$ & $-2 \cdot 6258$ \\
\hline & $0 \cdot 6$ & $-6 \cdot 3462$ & 4.0975 & $-2 \cdot 2487$ \\
\hline & $0 \cdot 4$ & -8.6796 & $8 \cdot 2178$ & -0.4618 \\
\hline & $0 \cdot 3$ & $-11 \cdot 1066$ & $14 \cdot 1795$ & $3 \cdot 0729$ \\
\hline \multirow[t]{12}{*}{$\mathrm{Ne}$} & $10 \cdot 0$ & $-258 \cdot 1772$ & $128 \cdot 2573$ & -129.9199 \\
\hline & $8 \cdot 0$ & $-258 \cdot 1771$ & $128 \cdot 2572$ & -129.9199 \\
\hline & $6 \cdot 0$ & $-258 \cdot 1747$ & $128 \cdot 2544$ & $-129 \cdot 9203$ \\
\hline & $4 \cdot 0$ & $-258 \cdot 1778$ & $128 \cdot 2630$ & $-129 \cdot 9148$ \\
\hline & 3.0 & $-258 \cdot 2279$ & $128 \cdot 3548$ & $-129 \cdot 8731$ \\
\hline & $2 \cdot 0$ & $-258 \cdot 7100$ & $129 \cdot 2800$ & $-129 \cdot 4300$ \\
\hline & 1.5 & $-260 \cdot 4154$ & $132 \cdot 5628$ & $-127 \cdot 8526$ \\
\hline & $1 \cdot 0$ & -277.8467 & 158.6617 & $-119 \cdot 1850$ \\
\hline & 0.8 & $-292 \cdot 3612$ & $185 \cdot 9834$ & $-106 \cdot 3778$ \\
\hline & 0.6 & $-324 \cdot 3163$ & 246.9639 & $-77 \cdot 3524$ \\
\hline & $0 \cdot 4$ & $-434 \cdot 1629$ & 489.6910 & $55 \cdot 5281$ \\
\hline & $0 \cdot 3$ & $-554 \cdot 4364$ & $845 \cdot 8103$ & $291 \cdot 3738$ \\
\hline \multirow[t]{12}{*}{$\mathrm{Ne}^{+}$} & $10 \cdot 0$ & $-256 \cdot 5539$ & $126 \cdot 0408$ & $-130 \cdot 5131$ \\
\hline & $8 \cdot 0$ & $-256 \cdot 5539$ & $126 \cdot 0408$ & $-130 \cdot 5131$ \\
\hline & $6 \cdot 0$ & $-256 \cdot 5538$ & $126 \cdot 0407$ & $-130 \cdot 5131$ \\
\hline & $4 \cdot 0$ & $-256 \cdot 5560$ & $126 \cdot 0433$ & $-130 \cdot 5127$ \\
\hline & 3.0 & $-256 \cdot 5974$ & 126.0911 & $-130 \cdot 5063$ \\
\hline & $2 \cdot 0$ & $-257 \cdot 0275$ & 126.6993 & $-130 \cdot 3282$ \\
\hline & $1 \cdot 5$ & $-258 \cdot 7490$ & $129 \cdot 2318$ & $-129 \cdot 5172$ \\
\hline & $1 \cdot 0$ & $-266 \cdot 3955$ & $141 \cdot 6721$ & $-124 \cdot 7234$ \\
\hline & 0.8 & -277.6087 & $161 \cdot 7620$ & $-115 \cdot 8466$ \\
\hline & 0.6 & $-316 \cdot 3342$ & $222 \cdot 0182$ & $-94 \cdot 3160$ \\
\hline & $0 \cdot 4$ & $-425 \cdot 5261$ & 438.8206 & $13 \cdot 2945$ \\
\hline & $0 \cdot 3$ & $-544 \cdot 1743$ & $757 \cdot 7473$ & $213 \cdot 5730$ \\
\hline
\end{tabular}




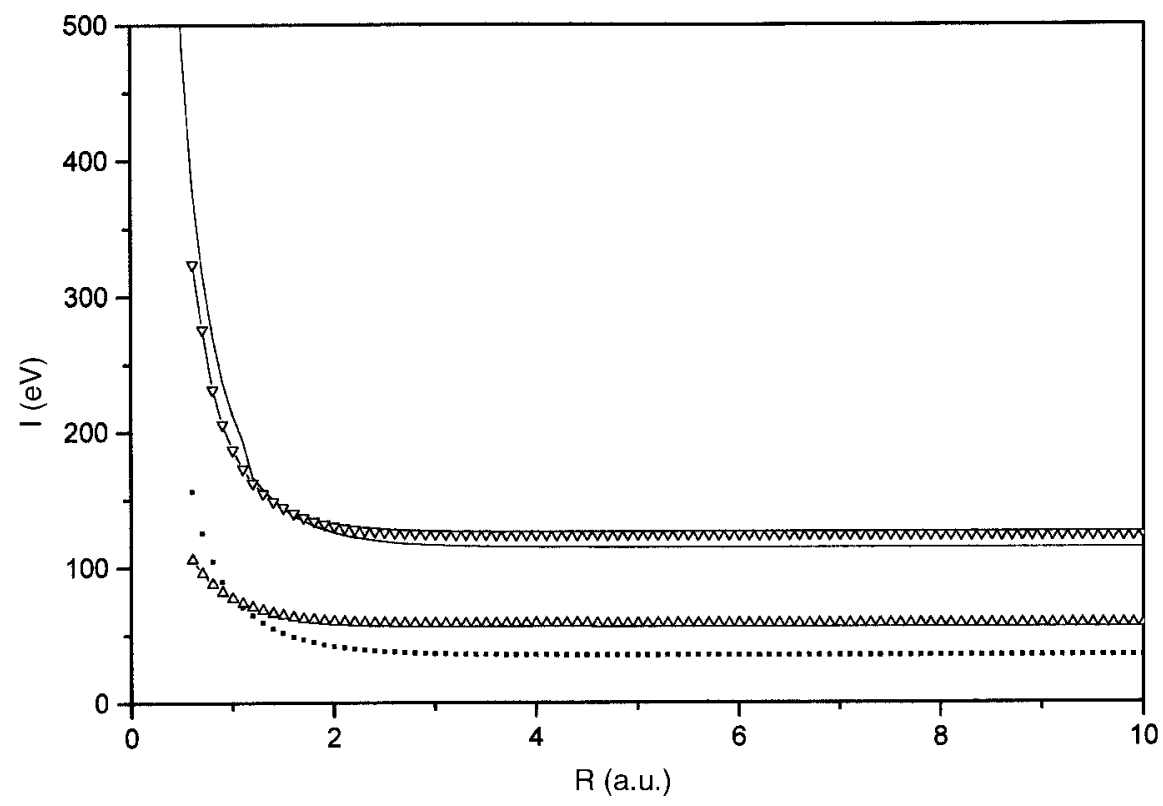

Figure 4. Plot of mean excitation energy $(I, \mathrm{eV})$ versus cut- off radius $(R$, a.u.) for $\mathrm{He}$ and $\mathrm{Ne}$ atoms and their ions confined in a spherical box. $(\cdots \cdots)$ He atom, $(-\triangle-)$ $\mathrm{He}^{+}$ion, $(-) \mathrm{Ne}$ atom, $(-\nabla-) \mathrm{Ne}^{+}$ion.

in excited state. In the departure regime, $\mu$ again changes drastically to reach a stable value more or less the same as at obtained in the approach regime.

The departure regime starts at $t=10.975$ a.u. onward, where we see again strong oscillations, indicating the charge readjustment and the return of electron density to the helium atom leaving the proton. Even after the proton moves a considerable distance away, the helium atom keeps on pulsating for quite some time. In the excited state, similar features are noticed.

Time evolution of the hardness is shown in figure 7. Hardness attains a maximum value in the encounter regime which may be considered to be a dynamical variant of the maximum hardness principle (MHP). The $\eta_{\max }$ values are shown in the table 3 with different projectile velocities, different impact parameters and different states. The $\eta_{\max }$ values for the ground state are greater than excited state and it increases with an increase in the projectile velocities and impact parameters, as expected from the MHP. It may be noted that here $\eta$ is calculated as a density functional and it does not require the ionization potential, electron affinity or orbital energy values per se. In the approach regime, $\eta$ remains more or less static. It suddenly increases and passes through a maximum in the encounter regime. The maximization of $\eta$ in the encounter regime and the larger $\eta_{\max }$ value for the ground state clearly reflect the validity of the maximum hardness principle in a dynamical context. In the departure regime, $\eta$ attains the same steady value as in the approach regime. With increasing the projectile velocity and impact parameter the value of the $\eta$ increases. 


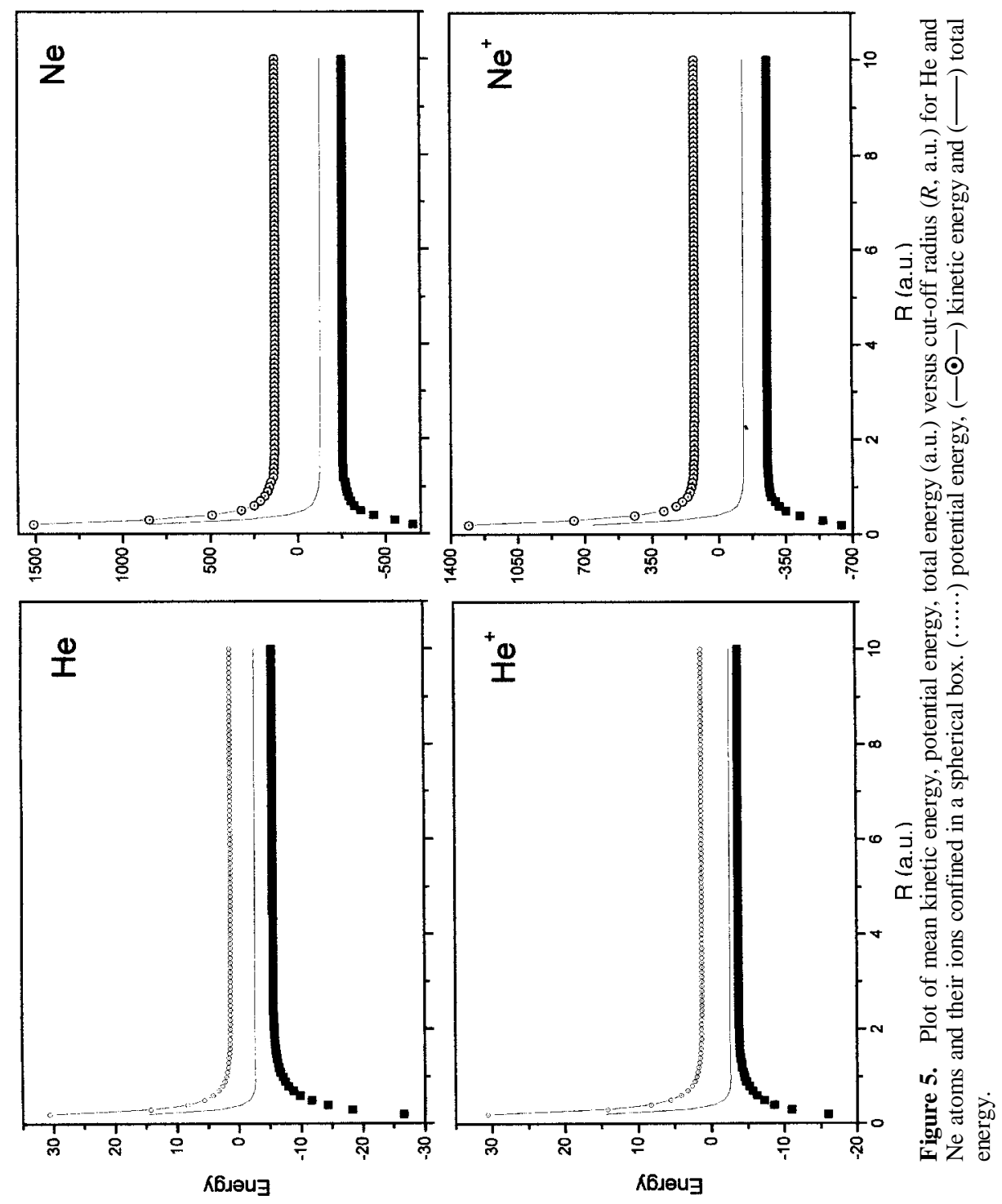


Table 2. Expectation values (in a.u.) of $\langle r\rangle,\left\langle r^{2}\right\rangle$ and $\langle 1 / r\rangle$ for different cut-off radii $(R$, a.u. $)$ for $\mathrm{He}$ and $\mathrm{Ne}$ atoms and their ions.

\begin{tabular}{|c|c|c|c|c|}
\hline Atom/ion & $R$ & $\langle r\rangle$ & $\left\langle r^{2}\right\rangle$ & $\langle 1 / r\rangle$ \\
\hline \multirow[t]{12}{*}{$\mathrm{He}$} & $10 \cdot 0$ & $0 \cdot 940246$ & $1 \cdot 233518$ & 1.692418 \\
\hline & $8 \cdot 0$ & $0 \cdot 940246$ & $1 \cdot 233514$ & 1.692418 \\
\hline & $6 \cdot 0$ & 0.940149 & $1 \cdot 232840$ & 1.692441 \\
\hline & $4 \cdot 0$ & 0.936164 & $1 \cdot 211827$ & 1.693617 \\
\hline & $3 \cdot 0$ & 0.917871 & $1 \cdot 135408$ & 1.701663 \\
\hline & $2 \cdot 0$ & 0.842262 & 0.898776 & 1.756841 \\
\hline & 1.5 & 0.744267 & 0.669505 & 1.876700 \\
\hline & 1.0 & $0 \cdot 581541$ & $0 \cdot 388410$ & $2 \cdot 224868$ \\
\hline & $0 \cdot 8$ & 0.490569 & $0 \cdot 271528$ & $2 \cdot 554247$ \\
\hline & 0.6 & 0.383892 & 0.163573 & $3 \cdot 162193$ \\
\hline & 0.4 & $0 \cdot 261344$ & 0.075227 & 4.572030 \\
\hline & 0.3 & 0.200234 & 0.043870 & $5 \cdot 884057$ \\
\hline \multirow[t]{12}{*}{$\mathrm{He}^{+}$} & $10 \cdot 0$ & 0.750006 & 0.750049 & $2 \cdot 000165$ \\
\hline & $8 \cdot 0$ & 0.750006 & 0.750049 & $2 \cdot 000165$ \\
\hline & $6 \cdot 0$ & 0.750005 & 0.750045 & 2.000165 \\
\hline & $4 \cdot 0$ & 0.749831 & 0.749259 & $2 \cdot 000266$ \\
\hline & 3.0 & 0.747358 & 0.740224 & 2.002134 \\
\hline & $2 \cdot 0$ & 0.720049 & 0.664792 & 2.032839 \\
\hline & 1.5 & 0.662078 & 0.539825 & $2 \cdot 127145$ \\
\hline & 1.0 & $0 \cdot 538638$ & $0 \cdot 339324$ & 2.450310 \\
\hline & $0 \cdot 8$ & $0 \cdot 461439$ & $0 \cdot 244000$ & $2 \cdot 770124$ \\
\hline & $0 \cdot 6$ & 0.366936 & 0.151201 & 3.365776 \\
\hline & $0 \cdot 4$ & 0.260622 & 0.074860 & $4 \cdot 588757$ \\
\hline & $0 \cdot 3$ & $0 \cdot 199988$ & 0.043775 & $5 \cdot 893574$ \\
\hline \multirow[t]{12}{*}{$\mathrm{Ne}$} & $10 \cdot 0$ & 0.766285 & 0.894939 & $3 \cdot 171599$ \\
\hline & $8 \cdot 0$ & $0 \cdot 766285$ & $0 \cdot 894937$ & $3 \cdot 171599$ \\
\hline & $6 \cdot 0$ & 0.766226 & $0 \cdot 894497$ & $3 \cdot 171607$ \\
\hline & $4 \cdot 0$ & 0.764159 & $0 \cdot 882862$ & $3 \cdot 172010$ \\
\hline & $3 \cdot 0$ & 0.755831 & $0 \cdot 845805$ & $3 \cdot 174471$ \\
\hline & $2 \cdot 0$ & 0.719130 & 0.723767 & $3 \cdot 192610$ \\
\hline & 1.5 & 0.664639 & 0.586512 & $3 \cdot 240234$ \\
\hline & $1 \cdot 0$ & 0.514937 & $0 \cdot 332243$ & $3 \cdot 565481$ \\
\hline & $0 \cdot 8$ & 0.442907 & 0.239045 & 3.831170 \\
\hline & $0 \cdot 6$ & 0.359049 & $0 \cdot 152396$ & $4 \cdot 324632$ \\
\hline & 0.4 & $0 \cdot 246313$ & $0 \cdot 071268$ & $5 \cdot 948437$ \\
\hline & $0 \cdot 3$ & $0 \cdot 180601$ & 0.038165 & $7 \cdot 731242$ \\
\hline \multirow[t]{12}{*}{$\mathrm{Ne}^{+}$} & $10 \cdot 0$ & 0.693915 & 0.716706 & 3.424285 \\
\hline & $8 \cdot 0$ & 0.693915 & 0.716706 & 3.424285 \\
\hline & $6 \cdot 0$ & 0.693912 & 0.716688 & 3.424285 \\
\hline & $4 \cdot 0$ & 0.693576 & 0.714941 & $3 \cdot 424372$ \\
\hline & 3.0 & 0.690648 & 0.702866 & 3.425612 \\
\hline & $2 \cdot 0$ & $0 \cdot 668234$ & 0.632051 & 3.438727 \\
\hline & 1.5 & 0.625324 & 0.527982 & 3.481505 \\
\hline & $1 \cdot 0$ & $0 \cdot 527875$ & $0 \cdot 351825$ & 3.645816 \\
\hline & 0.8 & 0.458432 & 0.257318 & 3.866957 \\
\hline & 0.6 & $0 \cdot 354716$ & $0 \cdot 150316$ & 4.490940 \\
\hline & 0.4 & 0.241923 & 0.069564 & $6 \cdot 186403$ \\
\hline & $0 \cdot 3$ & $0 \cdot 176244$ & $0 \cdot 036761$ & $8 \cdot 045274$ \\
\hline
\end{tabular}



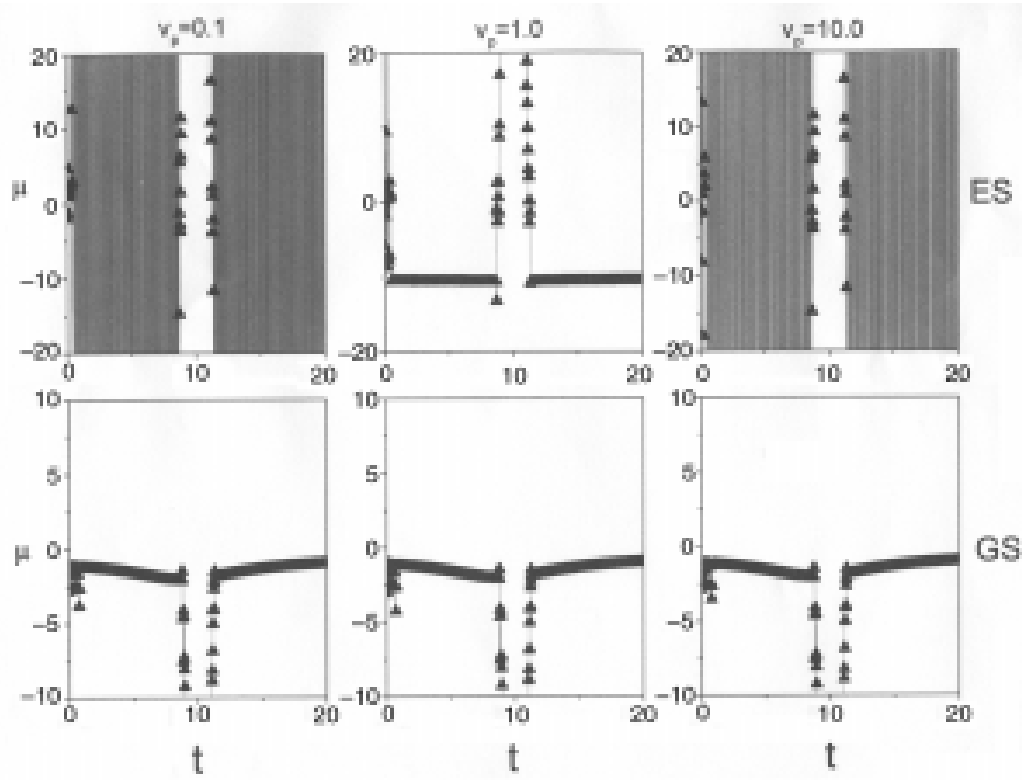

Figure 6. Time (a.u.) evolution of chemical potential ( $\mu$, a.u.) during a collision process between a $\mathrm{He}$ atom and a proton $(G S$, ground state; $E S$, excited state): $\mathrm{v}_{p}=0 \cdot 1,1 \cdot 0,10 \cdot 0 ;(-) b=0 \cdot 6,(\mathbf{\Delta} \mathbf{\Delta} \mathbf{\Delta}) b=1 \cdot 2$.
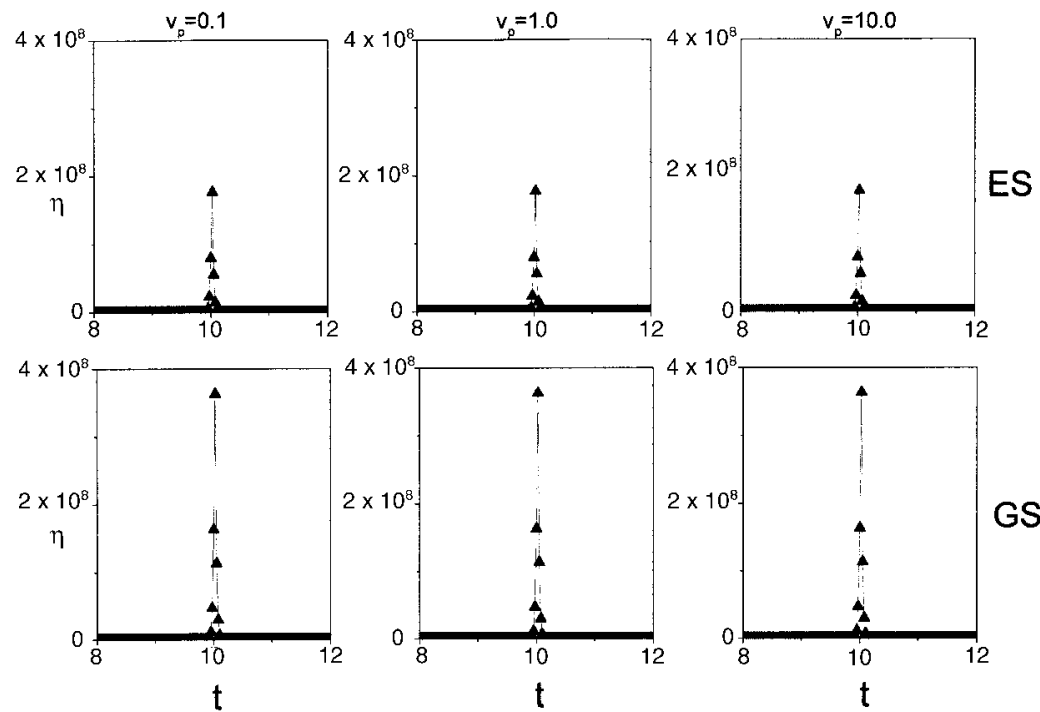

Figure 7. Time (a.u.) evolution of hardness ( $\eta$, a.u.) during a collision process between a $\mathrm{He}$ atom and a proton $\left(G S\right.$, ground state; $E S$, excited state): $\mathrm{v}_{p}=0 \cdot 1,1 \cdot 0$, $10 \cdot 0 ;(-) b=0.6,(\boldsymbol{\Delta} \boldsymbol{\Delta} \boldsymbol{\Delta}) b=1 \cdot 2$. 

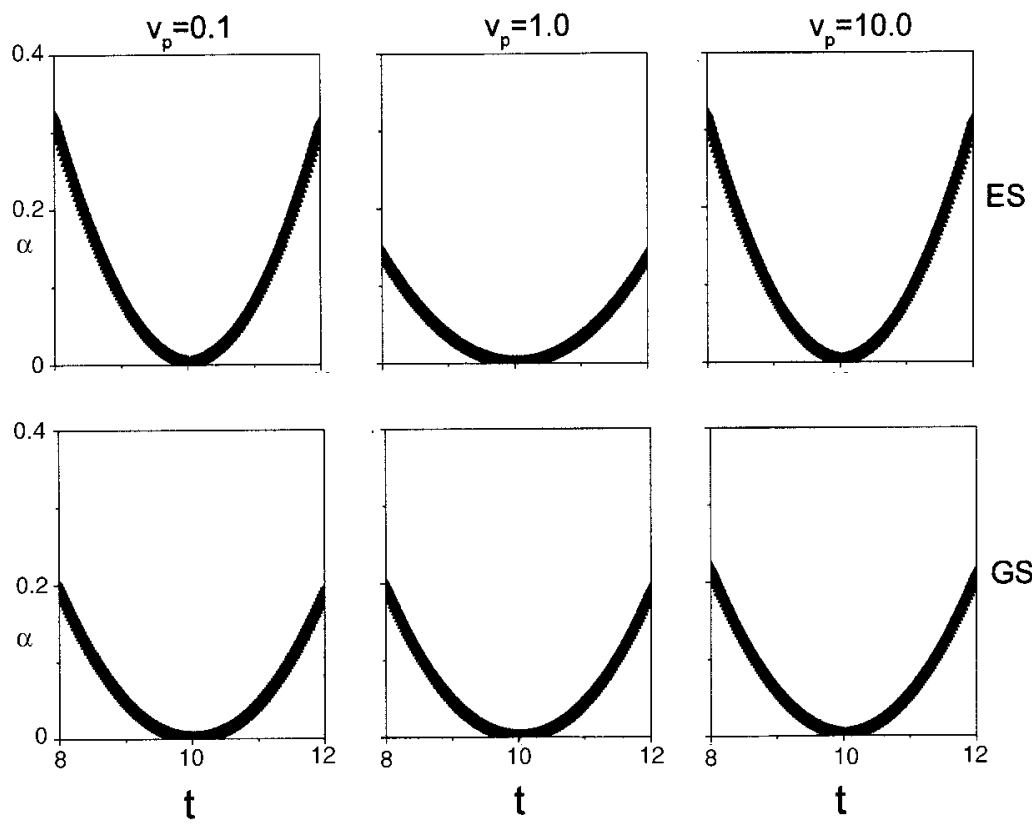

Figure 8. Time (a.u.) evolution of polarizability ( $\alpha$, a.u.) during a collision process between a He atom and a proton $\left(G S\right.$, ground state; $E S$, excited state): $\mathrm{v}_{p}=0 \cdot 1,1 \cdot 0$, $10 \cdot 0 ;(-) b=0 \cdot 6,(\mathbf{\Delta} \Delta \mathbf{\Delta}) b=1 \cdot 2$.

Table 3. The $\eta_{\max }, \alpha_{\min }, S_{\max }$ and $V_{p s}$ values at the closest approach during the collision between He atoms in the ground and excited states and protons $\left(\mathrm{V}_{p}=0 \cdot 1\right.$, $1 \cdot 0,10 \cdot 0 ; b=0 \cdot 6,1 \cdot 2)$. All units are atomic units.

\begin{tabular}{|c|c|c|c|c|}
\hline & \multicolumn{2}{|c|}{$b=0 \cdot 6$} & \multicolumn{2}{|c|}{$b=1 \cdot 2$} \\
\hline & $G S$ & $E S$ & $G S$ & $E S$ \\
\hline \multicolumn{5}{|c|}{$v_{p}=0 \cdot 1$} \\
\hline$\eta_{\max }$ & $3.62700 \times 10^{8}$ & $1.76870 \times 10^{8}$ & $3.62730 \times 10^{8}$ & $1.76873 \times 10^{8}$ \\
\hline$\alpha_{\min }$ & $7 \cdot 58761 \times 10^{-5}$ & $2.77000 \times 10^{-4}$ & $7 \cdot 58751 \times 10^{-5}$ & $2.66700 \times 10^{-4}$ \\
\hline$S_{\max }$ & 57.87625 & 48.05389 & $57 \cdot 87627$ & 48.05689 \\
\hline$V_{p s}$ & $10 \cdot 11640$ & $36 \cdot 67314$ & $10 \cdot 22740$ & $36 \cdot 82273$ \\
\hline \multicolumn{5}{|c|}{$v_{p}=1.0$} \\
\hline$\eta_{\max }$ & $3.62700 \times 10^{8}$ & $1.76872 \times 10^{8}$ & $3.62750 \times 10^{8}$ & $1.76875 \times 10^{8}$ \\
\hline$\alpha_{\min }$ & $7 \cdot 58447 \times 10^{-5}$ & $2.54218 \times 10^{-4}$ & $7 \cdot 58441 \times 10^{-5}$ & $2.54217 \times 10^{-4}$ \\
\hline$S_{\max }$ & $57 \cdot 87780$ & $49 \cdot 62760$ & $57 \cdot 87790$ & $49 \cdot 62800$ \\
\hline$V_{p s}$ & $10 \cdot 13710$ & $6175 \cdot 56150$ & $10 \cdot 23710$ & $6818 \cdot 17770$ \\
\hline \multicolumn{5}{|c|}{$v_{p}=10 \cdot 0$} \\
\hline$\eta_{\max }$ & $3.62740 \times 10^{8}$ & $1.76875 \times 10^{8}$ & $3.62770 \times 10^{8}$ & $1.76877 \times 10^{8}$ \\
\hline$\alpha_{\min }$ & $1 \cdot 15000 \times 10^{-5}$ & $2 \cdot 07000 \times 10^{-4}$ & $1.05000 \times 10^{-5}$ & $2.17000 \times 10^{-4}$ \\
\hline$S_{\max }$ & $57 \cdot 87928$ & 49.72670 & $57 \cdot 895280$ & $49 \cdot 82270$ \\
\hline$V_{p s}$ & $10 \cdot 13910$ & $19204 \cdot 10200$ & $10 \cdot 38110$ & $19870 \cdot 74000$ \\
\hline
\end{tabular}



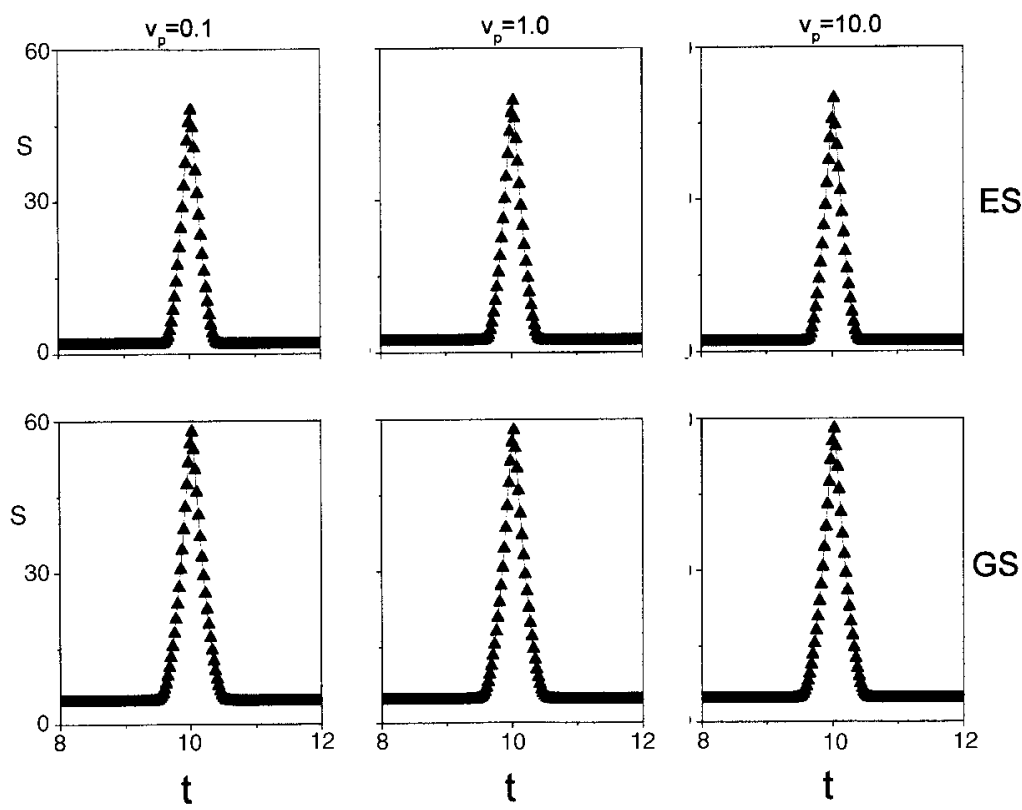

Figure 9. Time (a.u.) evolution of entropy ( $S$, a.u.) during a collision process between a $\mathrm{He}$ atom and proton $\left(G S\right.$, ground state; $E S$, excited state): $\mathrm{v}_{p}=0 \cdot 1,1 \cdot 0$, $10 \cdot 0 ;(-) b=0 \cdot 6,(\boldsymbol{\Delta} \boldsymbol{\Delta} \mathbf{\Delta}) b=1 \cdot 2$.
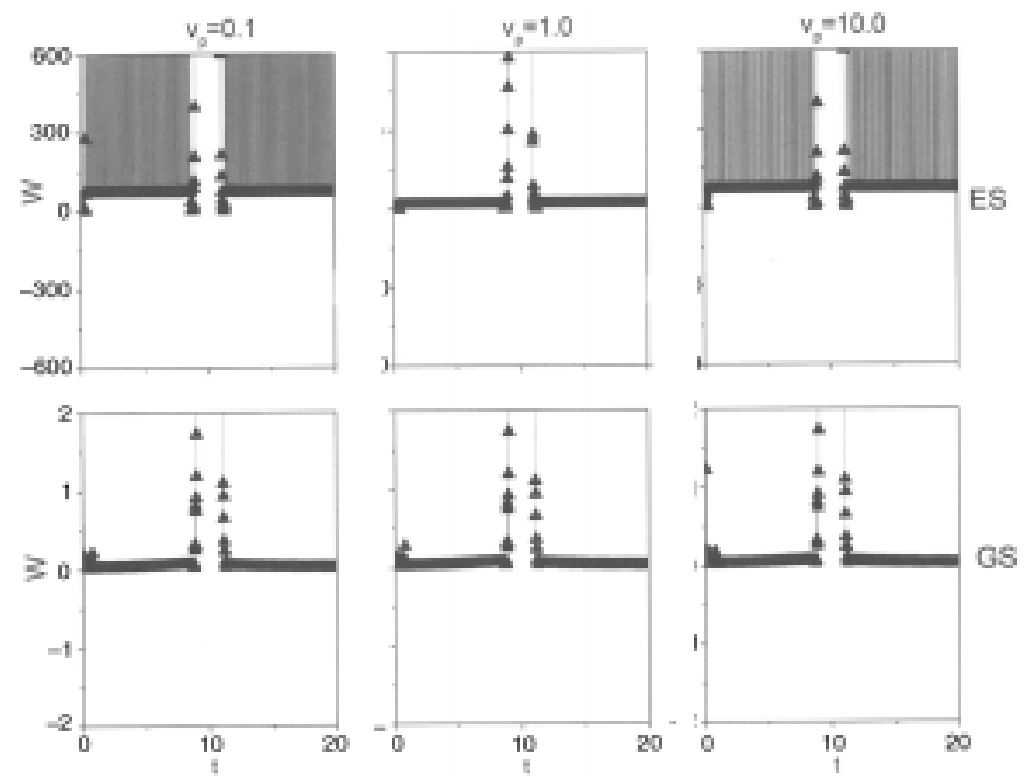

Figure 10. Time (a.u.) evolution of electrophilicity index ( $W$, a.u.) during a collision process between a He atom and proton $\left(G S\right.$, ground state; $E S$, excited state): $\mathrm{v}_{p}=0 \cdot 1$, $1 \cdot 0,10 \cdot 0 ;(-) b=0.6,(\mathbf{\Delta} \mathbf{\Delta} \mathbf{\Delta}) b=1 \cdot 2$. 
Figure 8 presents the time evolution of the dynamical polarizability. We notice that $\alpha$ becomes minimum in the encounter regime and a smaller $\alpha$ value for the ground state than for the excited state which is in conformity with the minimum polarizability principle (MPP). The $\alpha_{\min }$ values for the ground and excited states are shown in the table 3 with different projectile velocities and different impact parameters. It may be noted that the $\alpha_{\min }$ values of the ground state are less than those of the excited state with an increase in the projectile velocity and the impact parameter as expected from the MPP. Once the initial transients die out, $\alpha$ gradually decreases and passes through a minimum in the encounter regime when the two nuclei come closest to each other, as expected. The $\alpha_{\text {min }}$ values decrease as $\mathrm{b}$ and $v_{p}$ increase.

Figure 9 presents the time-dependent entropy. Once the initial undulations disappear, $S$ attains a steady value at the approach regime. In the encounter regime, it suddenly increases and passes through a maximum. In the departure regime $S$ attains the same steady value as in the approach regime. The maximum entropy principle reveals itself in these findings. The $S_{\max }$ values for the ground state and excited state are given in table 3 for different projectile velocities, different impact parameters and different states. The value of $S_{\max }$ increases with increasing projectile velocity and impact parameter in both ground and excited states. The values of $S$ in the ground state are greater than those of excited state.
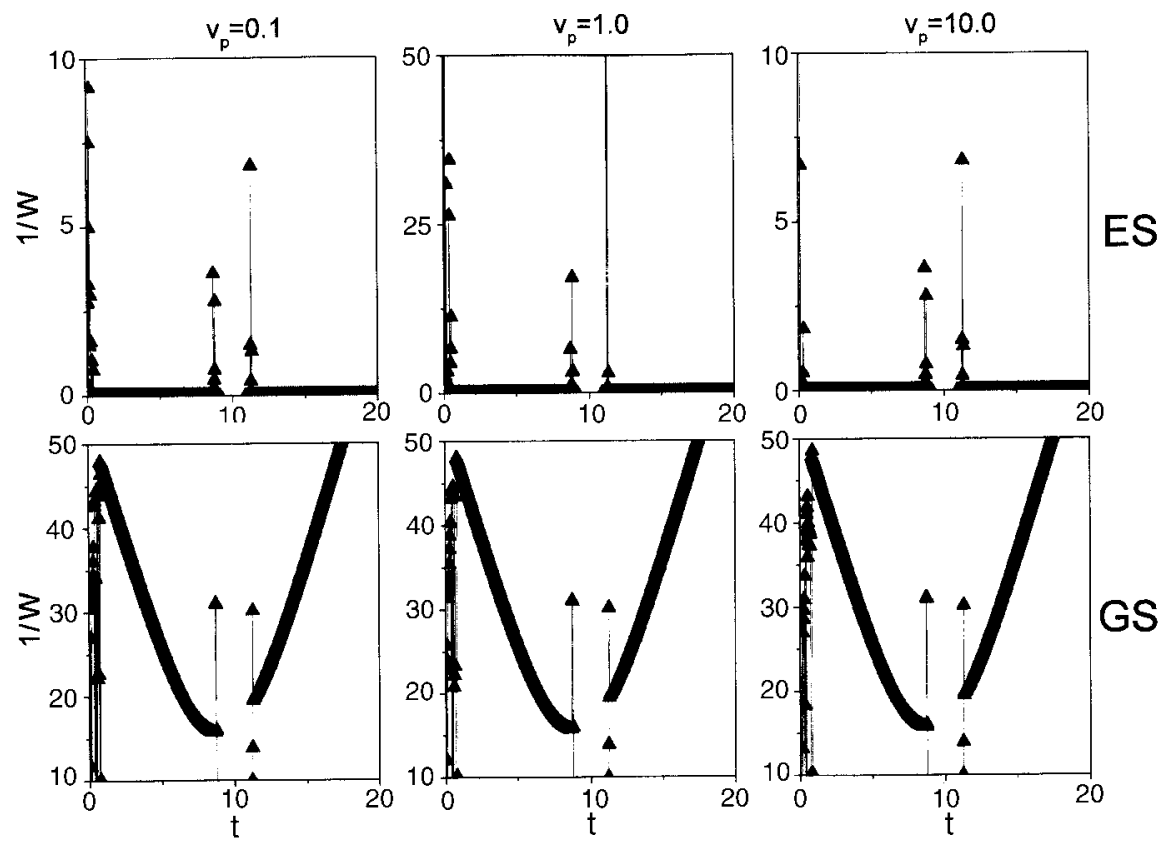

Figure 11. Time (a.u.) evolution of nucleophilicity index (1/W, a.u.) during a collision process between a He atom and proton ( $G S$, ground state; $E S$, excited state): $\mathrm{v}_{p}=0 \cdot 1,1 \cdot 0,10 \cdot 0 ;(-) b=0 \cdot 6,(\boldsymbol{\Delta} \mathbf{\Delta} \mathbf{\Delta}) b=1 \cdot 2$. 

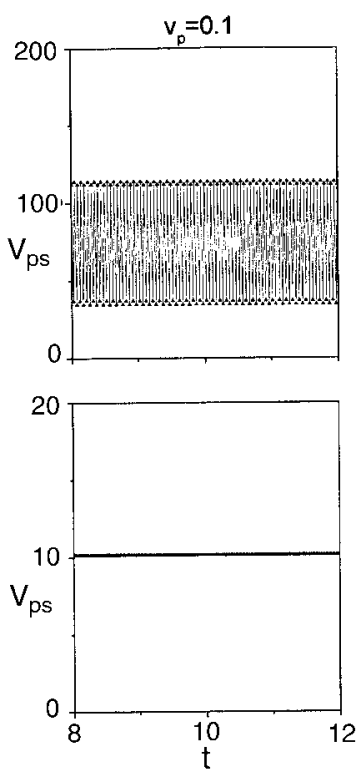
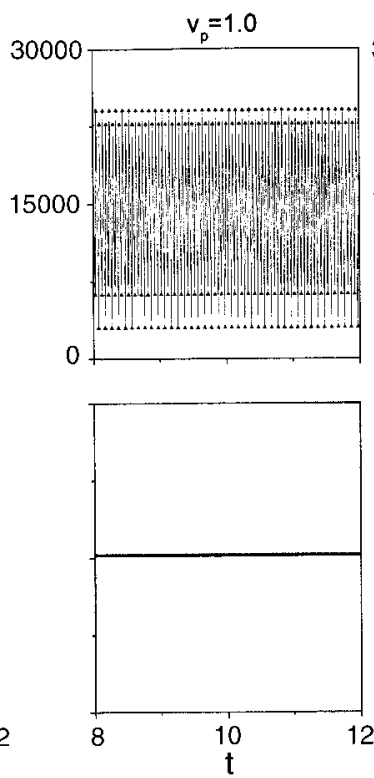

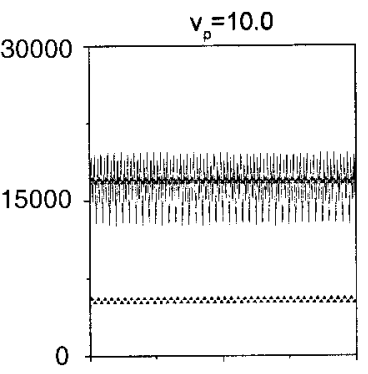

ES

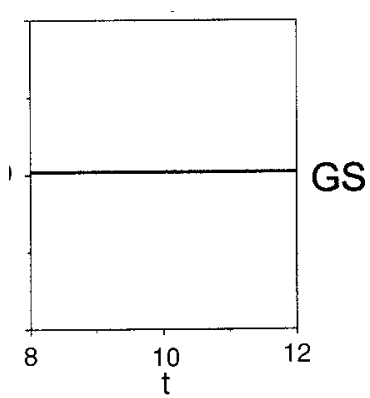

Figure 12. Time (a.u.) evolution of phase volume $\left(V_{p s}\right.$, a.u.) during a collision process between a He atom and proton $\left(G S\right.$, ground state; $E S$, excited state): $\mathrm{v}_{p}=0 \cdot 1$, $1 \cdot 0,10 \cdot 0 ;(-) b=0.6,(\boldsymbol{\Delta} \mathbf{\Delta} \Delta) b=1 \cdot 2$.

Figure 10 presents the dynamic profile of electrophilicity index $(W)$. The plots look like the plots of chemical potential $(\mu)$ for different projectile velocities and impact parameters in both ground and excited states.

Figure 11 presents the dynamic profile of nucleophilicty index $(1 / W)$. These plots are much simpler than the plots of chemical potential $(\mu)$ /electrophilicity index $(W)$.

Figure 12 shows the phase volume or the uncertainty product, $V_{p s}$. It confirms that the quantum fluctuations are always larger in the excited state than in the ground state as is expected from the more compactness of the ground state electron cloud. The values of $V_{p s}$ at the closest approach are listed in table 3 for different velocities, impact parameters and electronic states. $V_{p s}$ values increase with those of $b$ and $v_{p}$. A small portion of this work is published elsewhere. ${ }^{81}$

\section{Concluding remarks}

Various reactivity indices like softness, polarizability and mean excitation energy are studied for confined $\mathrm{He}$ and $\mathrm{Ne}$ atoms and their ions. This method shows that when the radius of the confining box increases, softness, polarizability and $\langle 1 / r\rangle$ increase but ionization energy $(I)$, total energy $(E),\langle r\rangle$ and $\left\langle r^{2}\right\rangle$ decrease. Increasing value of $I$ mimics the fact that more energy is needed for excitation which is also supported by the decreasing nature of the softness and polarizability. As expected, $\mathrm{He}^{+}$and $\mathrm{Ne}^{+}$are harder and less polarizable with larger excitation energies compared to their neutral atom counterparts. Therefore this method provides new physical insights into the problem of reactivity indices as well as total energy of the compressed systems. 
On the other hand, a quantum fluid density functional approach is found to be adequate in understanding the dynamics of ground- and excited-state electron densities of a helium atom interacting with an incoming proton. Dynamics of various reactivity parameters like electronegativity, hardness, polarizability, entropy, electrophilicity and nueleophilicity indices and phase space volume are studied. The whole collision process can be divided into approach, encounter and departure regimes in terms of the time-dependent chemical potential profile. In the encounter regime where the actual chemical process takes place, hardness and entropy maximize and polarizability minimizes. Dynamical variants of the maximum hardness principle, maximum entropy principle and minimum polarizability principle are observed to be valid for both electronic states and different values of impact parameters and projectile velocities.

\section{Acknowledgements}

We thank Prof. S S Krishnamurthy for kindly inviting us to publish the paper in this journal, the Council of Scientific and Industrial Research, New Delhi for financial support and the referee for constructive criticism.

\section{References}

1. Michels A, de Boer J and Bijl A 1937 Physica 4981

2. Sommerfeld A and Welker H 1928 Ann. Phys. 3256

3. de Groot S R and ten Seldam C A 1946 Physica 12669

4. ten Seldam C A and de Groot S R 1952 Physica 18891

5. Cottrell T L 1951 Trans. Faraday Soc. 4737

6. Gimarc B M 1966 J. Chem. Phys. 44373

7. Suryanarayana D and Weil J A 1976 J. Chem. Phys. 64510

8. Ley-Koo E and Rubinstein S 1979 J. Chem. Phys. 71351

9. Fernandez F M and Castro E A 1982 Int. J. Quantum Chem. 21741

10. Arteca G A, Fernandez F M and Castro E A 1984 J. Chem. Phys. 801569

11. Fröman P O, Yngve S and Fröman N 1987 J. Math. Phys. 821813

12. Yngve S J 1988 J. Math. Phys. 29931

13. Gorecki J and Byers B W 1987 J. Phys. B20 5953

14. Goodfriend P L 1990 J. Phys. B23 1373

15. Marin J L and Cruz S A 1991 J. Phys. B24 2899

16. Marin J L and Cruz S A 1991 Am. J. Phys. 59931

17. Goldman S and Joslin C 1992 J. Phys. Chem. 966021

18. Brownstein K R 1993 Phys. Rev. Lett. 711427

19. Dutt R, Mukherjee A and Varshni Y P 1995 Phys. Rev. A. 521750

20. Jaskólski W 1996 Phys. Rep. 271 1, and references therein

21. Ley-Koo E and Flores-Flores A 1998 Int. J. Quantum Chem. 66123

22. Luden̆a E V 1978 J. Chem. Phys. 691770

23. Ludeňa E V and Gregori M 1979 J. Chem. Phys. 712235

24. Gorecki J and Byers Brown W 1988 J. Phys. B21 403

25. LeSar R and Herschbach D R 1981 J. Phys. Chem. 852804

26. LeSar R and Herschbach D R 1983 J. Phys. Chem. 875202

27. Marin J L and Cruz S A 1992 J. Phys. B25 4365

28. Marin J L and Cruz S A 1995 J. Mol. Struct. (Theochem.) 287281

29. Marin J L, Rosas R and Uribe A 1995 Am. J. Phys. 63460

30. Corella-Madueňo A, Rosas R, Marin J L and Riera R 1999 Phys. Low Dim. Struct. 5/6 75

31. Parr R G and Yang W 1989 Density functional theory of atoms and molecules (New York Oxford University Press)

32. Haq S, Chattaraj P K and Deb B M 1984 Chem. Phys. Lett. 11179

33. Dirac P A M 1930 Proc. Cambridge Phil. Soc. 26376 
34. Brual G and Rothstein S M 1978 J. Chem. Phys. 691177

35. Sen K D and Jorgensen C K (eds) 1987 Electronegativity, structure and bonding (Berlin: Springer-Verlag) vol 66

36. Pearson R G 1997 Chemical hardness: Application form molecules to solids (Weinheim: Wiley-VCH Verlag)

37. Sen K D (eds) 1993 Chemical hardness: Structure and bonding (Berlin: Springer-Verlag) vol 80

38. Pauling L 1960 The Nature of the chemical bond 3rd edn (Ithaca: Cornell University Press)

39. Pearson R G 1990 Coord. Chem. Rev. 100403

40. Pearson R G 1973 Hard and soft acids and bases (Stroudsberg, PA: Dowden, Hutchinson \& Ross)

41. Fukui K 1982 Science 218747

42. Berkowitz M, Ghosh S K and Parr R G 1999 J. Am. Chem. Soc. 1211922

43. Hohenberg P and Kohn W 1964 Phys. Rev. B136 864

44. Kohn W and Sham L G 1965 Phys. Rev. A140 1133

45. Kulander K C, Sandhya Devi K R and Koonin S E 1982 Phys. Rev. A25 2968

46. Rudd M E, Kim Y K, Madison D H and Gay T J 1992 Rev. Mod. Phys. 64441

47. Runge E and Gross E K U 1984 Phys. Rev. Lett. 52997

48. Madelung E 1926 Z. Phys. 40322

49. Dey B K and Deb B M 1995 Int. J. Quant. Chem. 56707

50. Dey B K and Deb B M 1998 Int. J. Quant. Chem. 70441

51. Dey B K and Deb B M 1997 Pramana J. Phys. 48 L849

52. Deb B M, Chattaraj P K and Mishra S 1991 Phys. Rev. A43 1248

53. Deb B M and Chattaraj P K 1989 Phys. Rev. A39 1696

54. Sanderson R T 1951 Science 114670

55. Chattaraj P K and Sengupta S 1996 J. Phys. Chem. 10016126

56. Jaynes E T 1963 In Statistical physics (ed.) K W Ford, Brandeis Lectures (New York: Benjamin) vol 3

57. Parr R G, Szentpaly L V and Liu S 1999 J. Am. Chem. Soc. 1211922

58. Chattaraj P K and Sengupta S 1999 J. Phys. Chem. A103 6122

59. Fuentealba P 1995 J. Chem. Phys. 1036571

60. Garza J and Robles J 1993 Phys. Rev. A47 2680

61. Ahlen S P 1980 Rev. Mod. Phys. 52121

62. Hồ M, Weaver D F Jr and Smith V H 1998 Phys. Rev. A57 4512

63. Ghosh S K and Deb B M 1994 J. Phys. B27 381

64. Parr R G 1988 J. Phys. Chem. 923060

65. Dey B K and Deb B M 1998 Int. J. Quantum Chem. 67251

66. Deb B M and Ghosh S K 1982 J. Chem. Phys. 77342

67. Bartolotti L J 1981 Phys. Rev. A24 1661

68. Politzer R P, Parr R G and Murphy D R 1983 J. Chem. Phys. 793859

69. Herman F and Skillman S 1963 Atomic structure calculations (Englewood Cliffs, NJ: Prentice Hall)

70. Boeyens J C A 1994 J. Chem. Soc., Faraday Trans. 903377

71. Clementi E and Roetti C 1974 At. Data Nucl. Data Tab. 14174

72. Mukherjee P K, Sengupta S and Mukherji A 1970 Int. J. Quant. Chem. 4139

73. Politzer P 1987 J. Chem. Phys. 861072

74. Ghanty T K and Ghosh S K 1993 J. Phys. Chem. 974951

75. Hati S and Datta D 1994 J. Phys. Chem. 9810451

76. Fuentealba P and Reyes O 1993 J. Mol. Struct. (Theochem.) 28265

77. Roy R K, Chandra A K and Pal S 1995 J. Mol. Struct. (Theochem.) 331261

78. Sabin P B and Sabin J R 2001 Int. J. Quantum Chem. 82277

79. Gimarc B M 1967 J. Chem. Phys. 475110

80. Fischer C F 1977 The Hartree-Fock method for atoms: A numerical approach (New York: Wiley-Interscience)

81. Chattaraj P K and Sarkar U 2003 Chem. Phys. Lett. 372805 